\title{
Hybrid density functional theory applied to magnetite: Crystal structure, charge order, and phonons
}

\author{
Andrew D. Rowan and Charles H. Patterson \\ School of Physics, Trinity College Dublin, Dublin 2, Ireland
}

L. V. Gasparov

Department of Chemistry and Physics, University of North Florida, St. John's Bluff Road South, Jacksonville, Florida 32224, USA

(Received 18 November 2008; revised manuscript received 3 April 2009; published 5 May 2009)

\begin{abstract}
The electronic structure and equilibrium structure of magnetite $\left(\mathrm{Fe}_{3} \mathrm{O}_{4}\right)$ in the high temperature cubic $F d \overline{3} m$ and low temperature monoclinic $P 2 / c$ unit cells have been computed using the Perdew-Wang generalized gradient approximation (GGA) to density functional theory (DFT) and the B3LYP hybrid density functional. The ground state for the GGA-DFT is an itinerant electron metallic state in the cubic unit cell and the ground state for the B3LYP functional is a charge ordered semiconducting state in the monoclinic unit cell. The equilibrium structure predicted by the B3LYP functional for $\mathrm{Fe}_{3} \mathrm{O}_{4}$ in the $P 2 / c$ unit cell has been calculated with lattice parameters fixed at values obtained in recent $\mathrm{x}$-ray diffraction work and with the lattice fully relaxed. Bond lengths obtained with lattice parameters fixed at experimental values are in excellent agreement with X-ray measurements [J. P. Wright et al., Phys. Rev. B 66, 214422 (2002)]. The degree of charge order, measured as disproportionation of charge on octahedral B sites, is considerably less than unity and in reasonable agreement with values from resonant $\mathrm{x}$-ray diffraction measurements. However, conduction electrons are found to be fully localized on B1 and B4 sites in orbitally ordered $t_{2 g}$ states. This shows that they are formally $\mathrm{Fe}^{2+}$ ions while Fe B2 and B3 sites are formally $\mathrm{Fe}^{3+}$ sites. Therefore Verwey's original conjecture regarding charge localization in $\mathrm{Fe}_{3} \mathrm{O}_{4}$ applies, even though the specific pattern of charge order is different. GGA-DFT and B3LYP density functionals were used to calculate phonons at the $\Gamma$ point of the Brillouin zone. Phonon frequencies predicted for these crystal structures are compared to frequencies from infrared conductivity and Raman scattering experiments. Charge ordering causes symmetry breaking of force constants on symmetry lowering from the cubic $F d \overline{3} m$ unit cell to the $P 2 / c$ unit cell. This produces frequency splitting of modes which are degenerate in the cubic unit cell and concentration of ion displacements in phonon eigenvectors on particular Fe octahedral B site chains, especially in the highest frequency bands.
\end{abstract}

DOI: 10.1103/PhysRevB.79.205103

PACS number(s): 78.30.-j, 71.15.Mb, 71.30.+h

\section{INTRODUCTION}

The crystal structure, charge, and orbital order of magnetite $\left(\mathrm{Fe}_{3} \mathrm{O}_{4}\right)$ have been studied using a wide range of experimental ${ }^{1-13}$ and theoretical ${ }^{14-23}$ methods since the discovery of the Verwey transition in $1939 .{ }^{1,2}$ Early refinements of the crystal structure of $\mathrm{Fe}_{3} \mathrm{O}_{4}$ below the Verwey transition temperature $\left(T_{V} \sim 120 \mathrm{~K}\right)$ include neutron diffraction work by Iizumi and Shirane ${ }^{24}$ which used a detwinned crystal, and a single crystal $\mathrm{x}$-ray study. ${ }^{25}$ The neutron diffraction study showed the structure to have a monoclinic $\sqrt{2} a_{c} \times \sqrt{2} a_{c}$ $\times 2 a_{c}$ supercell with space group $C c,{ }^{24}$ where $a_{c}[=8.3939 \AA$ (Ref. 8)] is the lattice parameter of the high temperature cubic $F d \overline{3} m$ phase. The most recent $\mathrm{x}$-ray and neutron scattering data ${ }^{8}$ provide a crystal structure for $\mathrm{Fe}_{3} \mathrm{O}_{4}$ below $T_{V}$ which may be used to test the abilities of modern density functionals to predict properties of complex systems which undergo phase transitions.

In this paper we report calculations of the electronic structure, crystal structure, and $\Gamma$ point phonons of $\mathrm{Fe}_{3} \mathrm{O}_{4}$ in the cubic and monoclinic unit cells using generalized gradient approximation (GGA)-density functional theory (DFT) or B3LYP hybrid DFT. Hybrid density functionals are so called because they contain a linear combination of density functional and Hartree-Fock (HF) approximations to the exchange potential. The B3LYP exchange-correlation potential is given in Eq. (1); it contains HF exchange with a weight $A=0.2$,

$$
\begin{aligned}
E_{x c}= & (1-A)\left(E_{x}^{\mathrm{LDA}}+B E_{x}^{\mathrm{Becke}}\right)+A E_{x}^{\mathrm{HF}} \\
& +(1-C) E_{c}^{\mathrm{VWN}}+C E_{c}^{\mathrm{LYP}} .
\end{aligned}
$$

$E_{x}^{\mathrm{LDA}}$ (Ref. 26) is the local density approximation (LDA) to exchange; $E_{x}^{\text {Becke }}$ is Becke's gradient corrected exchange functional; ${ }^{27} E_{c}^{\mathrm{LYP}}$ is the Lee-Yang-Parr approximation ${ }^{28}$ to the correlation functional; $E_{c}^{\mathrm{VWN}}$ (Ref. 29) is the Vosko-WilkNusair approximation ${ }^{29}$ to the electron correlation functional. $A, B$, and $C$ are weight parameters. Inclusion of HF exchange in a hybrid density functional may open a band gap in a calculation on an insulating oxide, for which DFT predicts no band gap; indeed, the band gap predicted by a hybrid density functional depends linearly and strongly on the weight of HF exchange used in the calculation, by about $0.1 \mathrm{eV} \%$ change in the value of $A .^{30}$ In the $G W$ approximation $^{31,32}$ the HF exchange potential is screened by the inverse dielectric function and the corresponding "weight" of HF exchange depends on the wave vector and electron energy of a particular electron state. The effect of varying the parameter $A$ about the value $A=0.2$ in the B3LYP functional, on band gap and charge order of $\mathrm{Fe}_{3} \mathrm{O}_{4}$, is investigated here. 


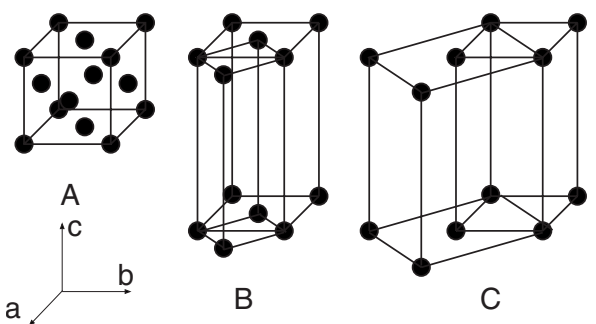

FIG. 1. (A) $F d \overline{3} m$ unit cell, (B) $P 2 / c$ unit cell, and (C) $C c$ unit cell for $\mathrm{Fe}_{3} \mathrm{O}_{4}$. Solid spheres indicate common vertices of unit cells rather than atomic positions.

The recent refinement of the crystal structure of $\mathrm{Fe}_{3} \mathrm{O}_{4},{ }^{8,33}$ above and below $T_{V}$, employed both neutron and synchrotron $\mathrm{X}$-ray powder-diffraction data at 130 and $90 \mathrm{~K}$. It was found that a smaller $P 2 / c$ unit cell than the full $C c$ cell could account for almost all diffraction peaks. ${ }^{8}$ The $C c$ unit cell contains 32 f.u. with 32 tetrahedral A sites and 64 octahedral $\mathrm{B}$ sites, of which $8 \mathrm{~A}$ and $16 \mathrm{~B}$ sites are unique. An NMR study $^{7}$ found spectral lines corresponding to all unique sites. Relationships between these unit cells are illustrated in Fig. 1. Figure 1(A) shows the cubic $F d \overline{3} m$ cell. The $P 2 / c$ cell is derived from the $F d \overline{3} m$ cell by doubling along the $c$ axis and reducing cell vector lengths by $1 / \sqrt{2}$ in the $a b$ plane of the $F d \overline{3} m$ cell [Fig. 1(B)]. The $C c$ cell is derived from the $P 2 / c$ cell by doubling in both $a$ and $b$ directions [Fig. 1(C)].

In the $F d \overline{3} m \mathrm{Fe}_{3} \mathrm{O}_{4}$ unit cell there are six $\mathrm{Fe}$ ions in two equivalent tetrahedral $\mathrm{A}$ sites and four equivalent octahedral $\mathrm{B}$ sites. All eight $\mathrm{O}$ ions are in equivalent sites. The primitive unit cell for the $P 2 / c$ phase (Fig. 1) contains eight $\mathrm{Fe}^{3+}$ ions with tetrahedral oxygen coordination in two crystallographic sites denoted $\mathrm{A} 1$ and $\mathrm{A} 2$, and $24 \mathrm{Fe}^{2+}$ and $\mathrm{Fe}^{3+}$ ions with octahedral coordination in six crystallographic sites denoted B1a, B1b, B2a, B2b, B3, and B4. The same notation is used here as was used by Wright et al. in Ref. 8. The arrangement of $\mathrm{Fe}$ ions in $\mathrm{B}$ sites consists of chains of B1 and B2 ions parallel to the $a$ lattice translation vector, connected by $\mathrm{O}$ ions with $90^{\circ} \mathrm{Fe}-\mathrm{O}-\mathrm{Fe}$ bond angles. There are similar mixed chains of B3 and B4 ions parallel to the $b$ vector. B1a and $\mathrm{B} 1 \mathrm{~b}$ and B2a and B2b ion pairs are almost crystallographically equivalent. ${ }^{8}$ It is convenient to think of the $P 2 / c$ unit cell of $\mathrm{Fe}_{3} \mathrm{O}_{4}$ in terms of chains of corner sharing $\mathrm{FeO}_{2}$ units, stacked in layers in the order B1, B3+B4, B2, B3+B4, connected by $\mathrm{Fe} \mathrm{A}$ site ions, and alternating in direction from layer to layer (Fig. 2). There are eight $\mathrm{O}$ sites in the $\mathrm{P} 2 / \mathrm{c}$ cell of $\mathrm{Fe}_{3} \mathrm{O}_{4}$, labeled O1-O4, O5a, O5b, O6a, and O6b. Ions labeled $\mathrm{a}$ and $\mathrm{b}$ are in nearly equivalent sites. Where both $\mathrm{a}$ and $b$ sites are referred to simultaneously in what follows, the $a, b$ designation of sites is omitted.

The inverse spinel electronic structure of magnetite, $\mathrm{Fe}^{3+}\left[\mathrm{Fe}^{2+} \mathrm{Fe}^{3+}\right] \mathrm{O}_{4}^{8-}$, admits the possibility of ordering of charges on octahedral $\mathrm{B}$ sites as $\mathrm{Fe}^{2+} 3 d^{6}$ and $\mathrm{Fe}^{3+} 3 d^{5}$ ions in a symmetry broken state below $T_{V}$. Charge localization and ordering on B sites were originally proposed by Verwey and Haayman ${ }^{2}$ to account for the 2 orders of magnitude reduction in electric conductivity when magnetite is cooled through $T_{V \cdot}{ }^{1,34}$ The Verwey model ${ }^{35}$ is one in which Coulomb repulsions of ordered $\mathrm{Fe}^{2+}$ and $\mathrm{Fe}^{3+}$ on octahedral $\mathrm{B}$

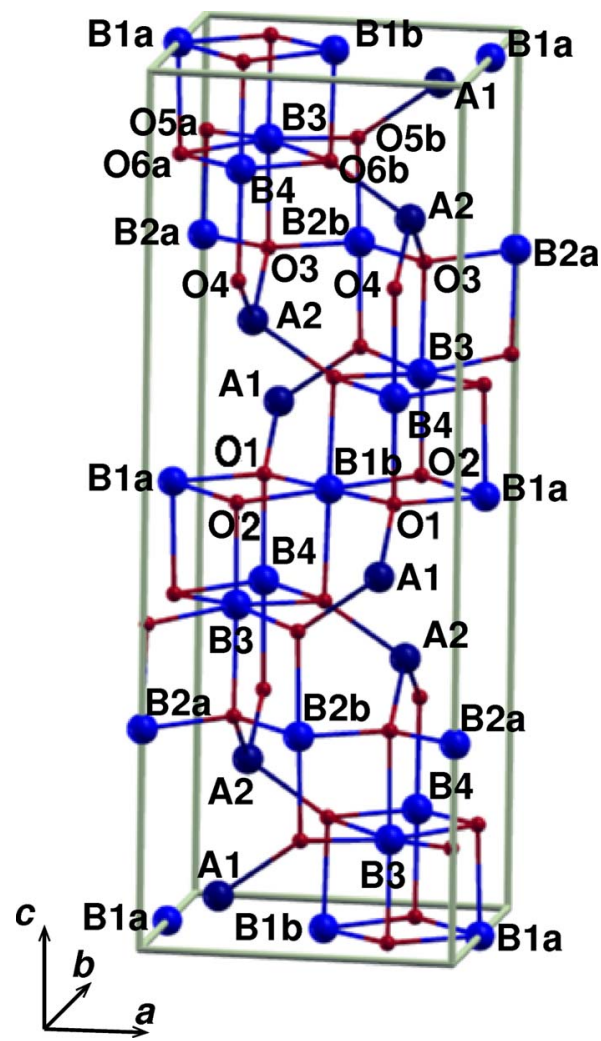

FIG. 2. (Color online) Primitive unit cell for $\mathrm{Fe}_{3} \mathrm{O}_{4}$ in the $P 2 / c$ unit cell. Octahedral Fe B site ions are shown as large light spheres, tetrahedral $\mathrm{Fe} \mathrm{A}$ site ions are shown as large dark spheres, and $\mathrm{O}$ ions are shown as small spheres. B1 and B2 site ions form separate chains parallel to the $a$ axis and B3 and B4 site ions form mixed chains parallel to the $b$ axis.

sites are minimized. Coulomb repulsion minimization was reduced to a simple criterion by Anderson, ${ }^{36}$ namely, that each Fe tetrahedron in the unit cell (formed, e.g., from two $\mathrm{B} 1$ ions in one chain and B3 and B4 ions in the chain beneath) should contain two $\mathrm{Fe}^{2+}$ and two $\mathrm{Fe}^{3+}$ ions. The pattern of charge order which has been found in first-principles calculations $^{15,18,19}$ is one where B1 and B4 sites are formally $\mathrm{Fe}^{2+}$ and B2 and B3 sites are formally $\mathrm{Fe}^{3+}$; Anderson's criterion $^{36}$ is not met by this charge order pattern. Experimental data from recent resonant $\mathrm{x}$-ray diffraction (RXD) studies $^{9,12,37}$ have been used to argue both for ${ }^{9,12}$ and against ${ }^{37}$ charge order in magnetite. RXD data were fitted to a model for charge ordering on Fe B sites which used charge disproportionation between $\mathrm{Fe} \mathrm{B} 1$ and $\mathrm{B} 2$ sites $\left(\delta_{12}\right)$ and between $\mathrm{B} 3$ and $\mathrm{B} 4$ sites $\left(\delta_{34}\right)$. The values found for $\delta_{12}$ and $\delta_{34}$ were $0.12 \pm 0.025 e$ and $0.10 \pm 0.06 e$, respectively. ${ }^{12} \delta$ $=0.5 e$ corresponds to complete disproportionation into $\mathrm{Fe}^{2+}$ and $\mathrm{Fe}^{3+}$ ions. Bond valence sums (BVSs) calculated for the structure determined by $\mathrm{x}$-ray diffraction were used to argue that the degree of charge disproportionation predicted on the basis of $\mathrm{B}$ site $\mathrm{Fe}-\mathrm{O}$ bond lengths ${ }^{8}$ is very similar to that found by RXD. ${ }^{9,12}$

Early measurements of the infrared (IR) vibrational spectrum of $\mathrm{Fe}_{3} \mathrm{O}_{4}$ were reported by Waldron in $1955 .{ }^{38}$ White and DeAngelis ${ }^{39}$ and Verble ${ }^{40}$ assigned the Raman spectrum of $\mathrm{Fe}_{3} \mathrm{O}_{4}$ and many subsequent studies of both the IR (Refs. 
41-43) and Raman spectra ${ }^{41-45}$ have been reported. Several calculations of the phonon modes of the $F d \overline{3} m$ cubic structure based on DFT have been reported recently. ${ }^{21,22,46}$ Here we report calculations of $\Gamma$ point phonon modes of both the cubic and monoclinic structures of $\mathrm{Fe}_{3} \mathrm{O}_{4}$ using either Perdew-Wang DFT-GGA (Ref. 47) or hybrid DFT functionals. The IR conductivity is calculated using a Lorentz oscillator (LO) model and compared to recent experimental data. ${ }^{42}$ Densities of phonon modes of appropriate symmetry are compared to Raman spectra. ${ }^{44}$ Phonon calculations for both structures were performed using the CRYSTAL electronic structure package ${ }^{48}$ and employed the same basis set and similar computational conditions. It is therefore possible to make a direct comparison between the phonon modes predicted by the two calculations.

The unit cell of the monoclinic $C c$ phase of $\mathrm{Fe}_{3} \mathrm{O}_{4}$ below $T_{V}$ contains 224 ions and contains too many ions for a phonon calculation using the CRYSTAL package on currently available computers. The unit cell of the $P 2 / c$ phase contains 56 ions; the phonon calculation for the smaller cell presented here is used to identify the character of the phonon modes which are observed in the low temperature phase of $\mathrm{Fe}_{3} \mathrm{O}_{4}$. A phonon calculation can also be used as a test of the stability of any equilibrium crystal structure since a saddle point on the potential energy surface manifests itself as an imaginary frequency of one or more phonon modes. Bond length distortions, which occur on going from the $F d \overline{3} m$ to the $P 2 / c$ phase, break site symmetries of $\mathrm{Fe}$ and $\mathrm{O}$ ions. Phonon mode frequencies, which are degenerate in the $F d \overline{3} m$ phase, split in the $P 2 / c$ phase and eigenvector amplitudes become concentrated on specific sites.

The equilibrium crystal structure of $\mathrm{Fe}_{3} \mathrm{O}_{4}$ found using both the GGA-DFT and B3LYP functionals is reported in Sec. II and compared to the structure found by $\mathrm{X}$-ray diffraction. ${ }^{8}$ The electronic structures of $\mathrm{Fe}_{3} \mathrm{O}_{4}$, obtained with either functional, are reported as well as charge and orbital order in the low temperature $P 2 / c$ unit cell. Results of calculations of phonons at the $\Gamma$ point are presented for the $F d \overline{3} m$ and $P 2 / c$ phases using the equilibrium crystal structures obtained using either the GGA-DFT or B3LYP functionals. In the discussion, the crystal structure of the $P 2 / c$ phase obtained from the B3LYP functional is compared with results of energy minimization $\mathrm{DFT}+U$ calculations. The charge order in the $P 2 / c$ phase is compared to that predicted by $\mathrm{DFT}+U$ or self-interaction corrected DFT (SICDFT) calculations; a brief discussion of application of hybrid density functional methods to transition metal oxides is given, followed by a discussion of the phonons and vibrational spectra of $\mathrm{Fe}_{3} \mathrm{O}_{4}$. Following the discussion and summary, details of calculations are given in the Appendix.

\section{RESULTS}

\section{A. Crystal structure}

Total energy minimization calculations were performed using GGA-DFT and B3LYP functionals. The high symmetry, high temperature $F d \overline{3} m$ structure is a stable energy minimum on the potential energy surface when a GGA-DFT functional is used; when the B3LYP functional is used with the cubic cell and $F d \overline{3} m$ space group symmetry constraints are relaxed, the energy minimized structure has no symmetry (space group $P_{1}$ ). When the B3LYP functional is used with the $P 2 / c$ cell the energy minimized structure closely resembles the structure obtained in recent $\mathrm{x}$-ray diffraction experiments. $^{8}$

Lattice parameters for the $P 2 / c$ unit cell from $\mathrm{x}$-ray diffraction data at $90 \mathrm{~K}$ are $a=5.944407, b=5.924701, c$ $=16.77515 \AA$, and $\beta=90.2363^{\circ}$ (Table II of Ref. 8). In this work the equilibrium structure of $\mathrm{Fe}_{3} \mathrm{O}_{4}$ in the $P 2 / c$ cell was calculated with the B3LYP functional, either by relaxing atomic positions with the lattice parameters fixed at these experimental values or by relaxing both atomic positions and lattice parameters simultaneously. These two structures are denoted below as "fixed" or "relaxed" according to whether atomic positions were relaxed with fixed or relaxed lattice parameters. Lattice parameters for the relaxed unit cell are $a=6.00788, b=6.01428, c=16.92349 \AA$, and $\beta=90.000$. These lattice parameters exceed the experimental values by $1.1 \%, 1.5 \%$, and $0.9 \%$, respectively.

Fractional coordinates of ions in the $P 2 / c$ cell from the B3LYP functional calculation as well as coordinates from the work of Wright et al. ${ }^{8}$ and Iizumi et al. ${ }^{49}$ are given in Table I. Mean absolute differences in fractional coordinates and corresponding displacements of ion positions are also given in Table I for the fixed cell calculation.

In order to obtain a stable structure refinement, Wright et $a l .{ }^{8}$ imposed additional Pmca pseudosymmetry constraints on Fe positions. Fe ions in B1a and B1b sites and in B2a and $\mathrm{B} 2 \mathrm{~b}$ sites were constrained to be equivalent. ${ }^{8}$ Since these pseudosymmetry constraints are not imposed in our calculations, it is possible to see the extent of symmetry breaking which occurs about Fe B1 and B2 sites when these constraints are absent. Bond lengths derived from experimental and energy minimization data in Table I are given in Table II. When bond lengths are obtained from our computed atomic positions, the maximum difference in $\mathrm{Fe}$ B1 and $\mathrm{B} 2$ site $\mathrm{Fe}-\mathrm{O}$ bond lengths is found to be $0.002 \AA$ (Table II) showing that the assumption of equivalent sites is valid.

Bond lengths in all unique $\mathrm{Fe} B$ site octahedra from a B3LYP functional energy minimization calculation, with lattice parameters fixed at values from $\mathrm{X}$-ray data, are shown in Fig. 3. The longest $\mathrm{B}$ site $\mathrm{Fe}-\mathrm{O}$ bonds are $\mathrm{B} 1 \mathrm{Fe}-\mathrm{O} 2$ and $\mathrm{B} 1$ $\mathrm{Fe}-\mathrm{O} 6, \mathrm{~B} 4 \mathrm{Fe}-\mathrm{O} 5$ and $\mathrm{B} 4 \mathrm{Fe}-\mathrm{O} 6$, and $\mathrm{B} 4 \mathrm{Fe}-\mathrm{O} 4$ and $\mathrm{B} 3$ $\mathrm{Fe}-\mathrm{O} 3$. The shortest $\mathrm{B}$ site $\mathrm{Fe}-\mathrm{O}$ bonds are all bonds to $\mathrm{Fe} \mathrm{B} 2$ and all bonds to Fe B3. When these are compared to bond lengths from $\mathrm{x}$-ray data refinement ${ }^{8}$ it is found that bonds to $\mathrm{Fe} \mathrm{B} 1$ except bonds to $\mathrm{O} 1$ are long, all bonds to Fe B2 are short, bonds to Fe B3 except bonds to O5 are short, and bonds to Fe B4 except bonds to $\mathrm{O} 1$ are long. There is good overall agreement between bond lengths from experiment and the B3LYP functional calculation for both A and B site $\mathrm{Fe}-\mathrm{O}$ bonds. Mean bond lengths from the B3LYP functional calculation, where lattice parameters were allowed to relax in the energy minimization calculation, are typically longer than those obtained with fixed lattice parameters by $\sim 0.02 \AA$. Long/short alternation of $\mathrm{Fe}-\mathrm{O}$ bond lengths in $\mathrm{B}$ site octahedra can be understood in terms of orientation of 
TABLE I. Fractional atomic coordinates for $\mathrm{Fe}_{3} \mathrm{O}_{4}$ from experiment by Wright et al. (Ref. 8) and Iizumi et al. (Ref. 49) (italics) and total energy minimization. The total energy was minimized with the lattice parameters fixed at their experimental values and with the lattice parameters relaxed simultaneously with fractional atomic coordinates. Data by Wright et al. (Ref. 8) and lizumi et al. (Ref. 49) were obtained using the $P 2 / c$ space group with Pmca pseudosymmetry constraints. Data from this work were obtained using the $P 2 / c$ space group with no pseudosymmetry constraints. Lattice parameters are $a=5.94441, b=5.92472, c=16.77508 \AA$, and $\beta=90.236^{\circ}$ from experiment (Ref. 8) and $a=6.00788, b=6.01428, c=16.92349 \AA$, and $\beta=90.000^{\circ}$ when lattice parameters were fully relaxed. Mean absolute differences in fractional coordinates between experiment and the fixed unit cell calculation and the corresponding mean absolute displacements of ions are given at the bottom of the table.

\begin{tabular}{|c|c|c|c|c|c|c|c|c|c|}
\hline \multirow[b]{2}{*}{ Atom } & \multicolumn{3}{|c|}{ Expt. } & \multicolumn{3}{|c|}{ Fixed } & \multicolumn{3}{|c|}{ Relaxed } \\
\hline & $x$ & $y$ & $z$ & $x$ & $y$ & $z$ & $x$ & $y$ & $z$ \\
\hline \multirow[t]{2}{*}{$\mathrm{A}(1)$} & $1 / 4$ & $0.0034(4)$ & $0.06366(7)$ & 0.24922 & 0.00743 & 0.06406 & 0.25000 & 0.00800 & 0.06367 \\
\hline & & $0.0049(3)$ & $0.0635(1)$ & & & & & & \\
\hline \multirow[t]{2}{*}{$\mathrm{A}(2)$} & $1 / 4$ & $0.5061(2)$ & $0.18867(8)$ & 0.25039 & 0.50335 & 0.18847 & 0.25000 & 0.50111 & 0.18873 \\
\hline & & $0.5067(2)$ & $0.1887(1)$ & & & & & & \\
\hline $\mathrm{B}(1 \mathrm{a})$ & 0 & $1 / 2$ & 0 & 0 & $1 / 2$ & 0 & 0 & $1 / 2$ & 0 \\
\hline $\mathrm{B}(1 \mathrm{~b})$ & $1 / 2$ & $1 / 2$ & 0 & $1 / 2$ & $1 / 2$ & 0 & $1 / 2$ & $1 / 2$ & 0 \\
\hline \multirow[t]{2}{*}{$\mathrm{B}(2 \mathrm{a})$} & 0 & $0.0096(3)$ & $1 / 4$ & 0 & 0.00652 & $1 / 4$ & 0 & 0.00422 & $1 / 4$ \\
\hline & & $0.0099(3)$ & & & & & & & \\
\hline \multirow[t]{2}{*}{$\mathrm{B}(2 \mathrm{~b})$} & $1 / 2$ & $0.0096(3)$ & $1 / 4$ & $1 / 2$ & 0.00647 & $1 / 4$ & $1 / 2$ & 0.00421 & $1 / 4$ \\
\hline & & $0.0099(3)$ & & & & & & & \\
\hline \multirow[t]{2}{*}{$\mathrm{B}(3)$} & $1 / 4$ & $0.2659(2)$ & $0.38010(9)$ & 0.25032 & 0.26244 & 0.37940 & 0.25000 & 0.25997 & 0.37917 \\
\hline & & $0.2643(4)$ & $0.3789(1)$ & & & & & & \\
\hline \multirow[t]{2}{*}{$\mathrm{B}(4)$} & $1 / 4$ & $0.7520(2)$ & $0.37659(9)$ & 0.25021 & 0.75225 & 0.37612 & 0.25000 & 0.75061 & 0.37608 \\
\hline & & $0.7549(5)$ & $0.3746(2)$ & & & & & & \\
\hline \multirow[t]{2}{*}{$\mathrm{O}(1)$} & $1 / 4$ & $0.2637(7)$ & $-0.0023(3)$ & 0.25008 & 0.26275 & -0.00222 & 0.25000 & 0.26313 & -0.00210 \\
\hline & & $0.2630(6)$ & $-0.0027(2)$ & & & & & & \\
\hline \multirow[t]{2}{*}{$\mathrm{O}(2)$} & $1 / 4$ & $0.7461(6)$ & $-0.0029(3)$ & 0.25007 & 0.75500 & -0.00369 & 0.25000 & 0.75627 & -0.00413 \\
\hline & & $0.7477(6)$ & $-0.0009(2)$ & & & & & & \\
\hline \multirow[t]{2}{*}{$\mathrm{O}(3)$} & $1 / 4$ & $0.2447(8)$ & $0.2542(3)$ & 0.24989 & 0.23864 & 0.25400 & 0.25000 & 0.23566 & 0.25380 \\
\hline & & $0.2461(7)$ & $0.2450(2)$ & & & & & & \\
\hline \multirow[t]{2}{*}{$\mathrm{O}(4)$} & $1 / 4$ & $0.7738(8)$ & $0.2525(3)$ & 0.24997 & 0.77398 & 0.25005 & 0.25000 & 0.77237 & 0.24972 \\
\hline & & $0.7696(6)$ & $0.2427(2)$ & & & & & & \\
\hline \multirow[t]{2}{*}{$\mathrm{O}(5 \mathrm{a})$} & $-0.0091(4)$ & $0.0095(6)$ & $0.1277(2)$ & -0.00851 & 0.01345 & 0.13092 & -0.00816 & 0.01224 & 0.13095 \\
\hline & $-0.0116(6)$ & $0.0089(3)$ & $0.1295(2)$ & & & & & & \\
\hline \multirow[t]{2}{*}{$\mathrm{O}(5 \mathrm{~b})$} & $0.4909(4)$ & $0.0095(6)$ & $0.3723(2)$ & 0.49202 & 0.01361 & 0.36899 & 0.49185 & 0.01224 & 0.36905 \\
\hline & $0.4884(6)$ & $0.0089(3)$ & $0.3705(2)$ & & & & & & \\
\hline \multirow[t]{2}{*}{$\mathrm{O}(6 \mathrm{a})$} & $-0.0081(4)$ & $0.5046(6)$ & $0.1246(2)$ & -0.00991 & 0.49338 & 0.12697 & -0.01066 & 0.49071 & 0.12729 \\
\hline & $-0.0067(6)$ & $0.5050(3)$ & $0.1244(1)$ & & & & & & \\
\hline \multirow[t]{2}{*}{$\mathrm{O}(6 \mathrm{~b})$} & $0.4919(4)$ & $0.5046(6)$ & $0.3754(2)$ & 0.49038 & 0.49367 & 0.37300 & 0.48934 & 0.49071 & 0.37271 \\
\hline & $0.4933(6)$ & $0.5050(3)$ & $0.3756(1)$ & & & & & & \\
\hline Difference & & & & 0.0013 & 0.0046 & 0.0046 & & & \\
\hline Displacement & & & & 0.008 & 0.027 & 0.077 & & & \\
\hline
\end{tabular}

ordered $t_{2 g}$ orbitals on $\mathrm{B} 1$ and $\mathrm{B} 4$ sites (see Sec. II C).

The crystal structure of $\mathrm{Fe}_{3} \mathrm{O}_{4}$ in the $F d \overline{3} m$ cell is uniquely specified by one lattice parameter and one $\mathrm{O}$ ion coordinate parameter, $x$, which specifies the $e$ Wyckoff position in the $F d \overline{3} m$ space group. The experimental lattice parameters are $8.3963 \AA$ and $x=0.25490$ (Ref. 8); the corre- sponding parameters derived from a B3LYP energy minimization calculation in which $F d \overline{3} m$ symmetry constraints were applied to the atomic positions are $8.48400 \AA$ and $x=0.25425$. This is not the ground state for the 14 atom unit cell of the cubic phase of $\mathrm{Fe}_{3} \mathrm{O}_{4}$ using a B3LYP functional, instead the B3LYP functional predicts a charge or- 
TABLE II. Fe-O bond lengths in $\AA$ for unique Fe ions derived from $\mathrm{x}$-ray diffraction data and B3LYP functional energy minimization calculations for structures given in Table I. Mean bond lengths for each unique $\mathrm{Fe}$ ion site are given in angular brackets.

\begin{tabular}{|c|c|c|c|}
\hline Bond & Expt. $^{a}$ & Fixed & Relaxed \\
\hline $\mathrm{A}(1)-\mathrm{O}(1)$ & $1.898(4)$ & 1.877 & 1.924 \\
\hline $\mathrm{A}(1)-\mathrm{O}(5 \mathrm{a})$ & $1.875(2)$ & 1.903 & 1.896 \\
\hline $\mathrm{A}(1)-\mathrm{O}(5 \mathrm{~b})$ & $1.882(2)$ & 1.901 & 1.900 \\
\hline $\mathrm{A}(1)-\mathrm{O}(2)$ & $1.890(4)$ & 1.879 & 1.924 \\
\hline$\langle\mathrm{A}(1)-\mathrm{O}\rangle$ & $1.886(3)$ & 1.890 & 1.911 \\
\hline $\mathrm{A}(2)-\mathrm{O}(4)$ & $1.913(5)$ & 1.907 & 1.939 \\
\hline$A(2)-O(6 a)$ & $1.870(2)$ & 1.857 & 1.881 \\
\hline$A(2)-O(6 b)$ & $1.877(2)$ & 1.859 & 1.881 \\
\hline $\mathrm{A}(2)-\mathrm{O}(3)$ & $1.899(5)$ & 1.915 & 1.931 \\
\hline$\langle\mathrm{A}(2)-\mathrm{O}\rangle$ & $1.890(4)$ & 1.885 & 1.908 \\
\hline $\mathrm{B}(1 \mathrm{a})-\mathrm{O}(1) \times 2$ & $2.042(3)$ & 2.046 & 2.070 \\
\hline $\mathrm{B}(1 \mathrm{a})-\mathrm{O}(6 \mathrm{a}) \times 2$ & $2.091(3)$ & 2.131 & 2.156 \\
\hline $\mathrm{B}(1 \mathrm{a})-\mathrm{O}(2) \times 2$ & $2.082(3)$ & 2.121 & 2.153 \\
\hline$\langle\mathrm{B}(1)-\mathrm{O}\rangle$ & $2.072(3)$ & 2.099 & 2.126 \\
\hline $\mathrm{B}(1 \mathrm{~b})-\mathrm{O}(1) \times 2$ & $2.042(3)$ & 2.045 & 2.070 \\
\hline $\mathrm{B}(1 \mathrm{~b})-\mathrm{O}(6 \mathrm{~b}) \times 2$ & $2.091(3)$ & 2.131 & 2.156 \\
\hline $\mathrm{B}(1 \mathrm{~b})-\mathrm{O}(2) \times 2$ & $2.082(3)$ & 2.120 & 2.153 \\
\hline$\langle\mathrm{B}(1)-\mathrm{O}\rangle$ & $2.072(3)$ & 2.099 & 2.126 \\
\hline $\mathrm{B}(2 \mathrm{a})-\mathrm{O}(5 \mathrm{a}) \times 2$ & $2.052(3)$ & 1.998 & 2.049 \\
\hline $\mathrm{B}(2 \mathrm{a})-\mathrm{O}(4) \times 2$ & $2.040(3)$ & 2.026 & 2.049 \\
\hline $\mathrm{B}(2 \mathrm{a})-\mathrm{O}(3) \times 2$ & $2.038(4)$ & 2.025 & 2.016 \\
\hline$\langle\mathrm{B}(2)-\mathrm{O}\rangle$ & $2.043(3)$ & 2.016 & 2.038 \\
\hline $\mathrm{B}(2 \mathrm{~b})-\mathrm{O}(5 \mathrm{~b}) \times 2$ & $2.052(3)$ & 1.997 & 2.049 \\
\hline $\mathrm{B}(2 \mathrm{~b})-\mathrm{O}(4) \times 2$ & $2.040(3)$ & 2.026 & 2.049 \\
\hline $\mathrm{B}(2 \mathrm{~b})-\mathrm{O}(3) \times 2$ & $2.038(4)$ & 2.027 & 2.016 \\
\hline$\langle\mathrm{B}(2)-\mathrm{O}\rangle$ & $2.043(3)$ & 2.016 & 2.038 \\
\hline $\mathrm{B}(3)-\mathrm{O}(5 \mathrm{a})$ & $2.092(3)$ & 2.066 & 2.088 \\
\hline $\mathrm{B}(3)-\mathrm{O}(5 \mathrm{~b})$ & $2.092(3)$ & 2.066 & 2.088 \\
\hline $\mathrm{B}(3)-\mathrm{O}(6 \mathrm{a})$ & $2.018(3)$ & 1.981 & 2.001 \\
\hline $\mathrm{B}(3)-\mathrm{O}(6 \mathrm{~b})$ & $2.018(3)$ & 1.981 & 2.001 \\
\hline $\mathrm{B}(3)-\mathrm{O}(2)$ & $1.964(5)$ & 1.964 & 1.977 \\
\hline $\mathrm{B}(3)-\mathrm{O}(3)$ & $2.116(5)$ & 2.108 & 2.127 \\
\hline$\langle\mathrm{B}(3)-\mathrm{O}\rangle$ & $2.050(4)$ & 2.027 & 2.047 \\
\hline $\mathrm{B}(4)-\mathrm{O}(5 \mathrm{a})$ & $2.094(3)$ & 2.115 & 2.145 \\
\hline $\mathrm{B}(4)-\mathrm{O}(5 \mathrm{~b})$ & $2.094(3)$ & 2.117 & 2.145 \\
\hline $\mathrm{B}(4)-\mathrm{O}(6 \mathrm{a})$ & $2.053(3)$ & 2.096 & 2.125 \\
\hline $\mathrm{B}(4)-\mathrm{O}(6 \mathrm{~b})$ & $2.053(3)$ & 2.095 & 2.125 \\
\hline $\mathrm{B}(4)-\mathrm{O}(1)$ & $2.033(5)$ & 2.043 & 2.063 \\
\hline $\mathrm{B}(4)-\mathrm{O}(4)$ & $2.086(5)$ & 2.119 & 2.145 \\
\hline$\langle\mathrm{B}(4)-\mathrm{O}\rangle$ & $2.069(4)$ & 2.098 & 2.125 \\
\hline
\end{tabular}

aReference 8 .

dered semiconducting state which breaks $F d \overline{3} m$ symmetry when symmetry constraints are relaxed. The cubic phase of $\mathrm{Fe}_{3} \mathrm{O}_{4}$ was also studied using a DFT-GGA functional; in this case the ground state is an itinerant electron metallic state
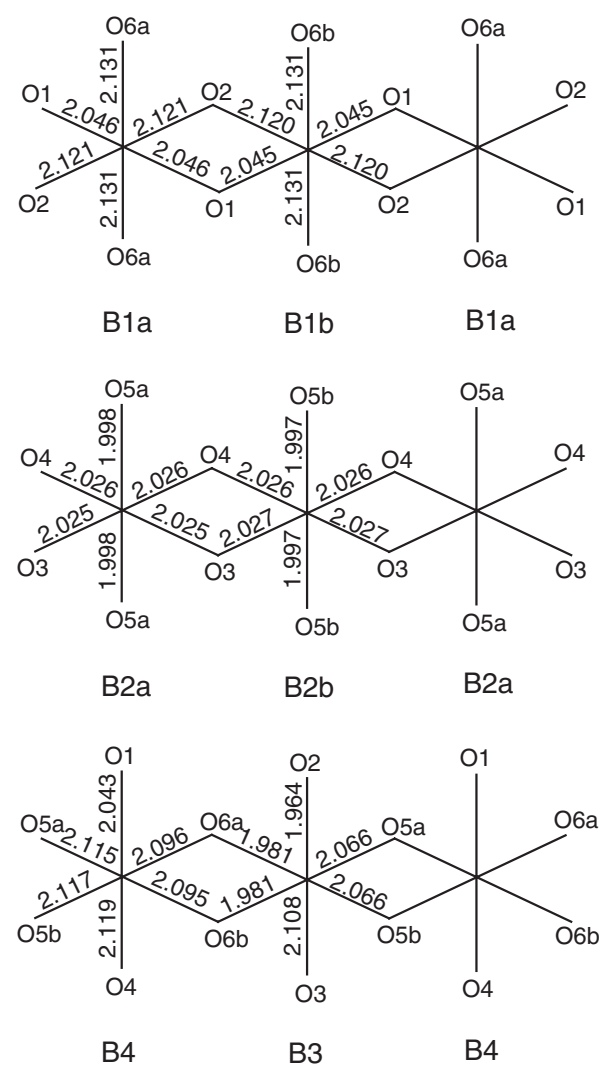

FIG. 3. Fe-O bond lengths around octahedral Fe sites from a B3LYP functional energy minimization calculation with lattice parameters fixed at values determined by $\mathrm{x}$-ray diffraction (Table I, fixed column).

with $F d \overline{3} m$ symmetry and equilibrium lattice constants, $a_{c}$ $=8.3777 \AA$ and $x=0.25499$.

\section{B. Electronic structure}

The $F d \overline{3} m$ space group and lattice for $\mathrm{Fe}_{3} \mathrm{O}_{4}$ above $T_{V}$ are the same as for the diamond structure, so they share the same familiar Brillouin zone. The band structure and density of states obtained using the DFT-GGA functional in the $F d \overline{3} m$ unit cell are shown in Figs. 4 and 5; no shift of energy eigenvalues has been applied to energy bands. The majority spin band gap is $3.6 \mathrm{eV}$ and the minority spin band structure has four bands occupied by two electrons close to the Fermi level at $-1.2 \mathrm{eV}$.

The majority spin band structure for $\mathrm{Fe}_{3} \mathrm{O}_{4}$ in the $P 2 / c$ unit cell (Fig. 6) has a band gap of $3.2 \mathrm{eV}$. The minority spin band structure has eight narrow filled bands around $-2 \mathrm{eV}$, which contain the valence band maximum. The band structure shown uses high symmetry points of the Brillouin zone for which wave vectors are parallel to the $a b$ plane of the crystal. There is little dispersion for wave vectors parallel to the long $c$ axis, as expected. The band gap is $0.87 \mathrm{eV}$ and the gap separates occupied states on Fe B1 and B4 sites from unoccupied states on B2 and B3 sites. Figure 7 shows the density of states projected onto B sites. Occupied majority spin $\mathrm{Fe}$ states are nearly exclusively B site states while oc- 


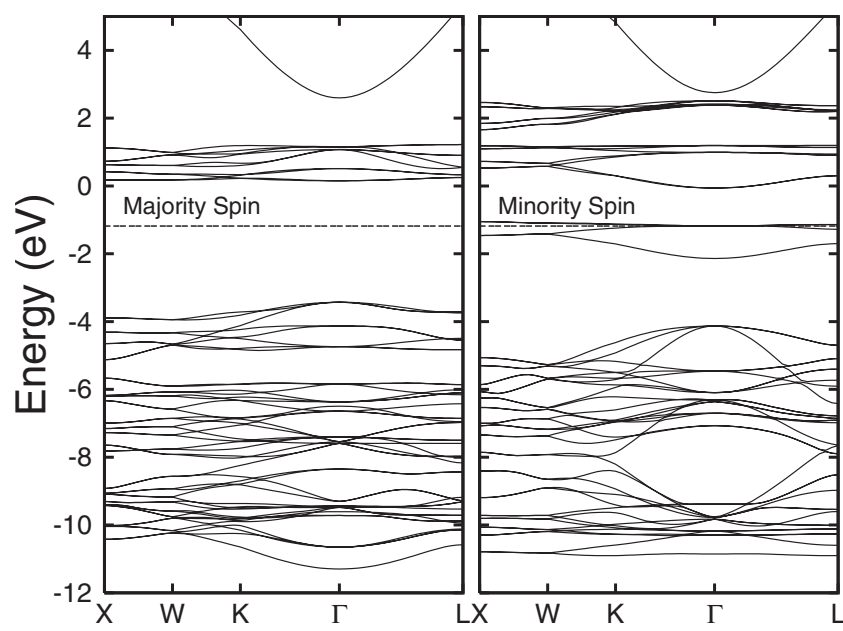

FIG. 4. Electronic band structure of magnetite in the $F d \overline{3} m$ unit cell. The Fermi energy is shown as a dashed line.

cupied minority spin Fe states (not shown) are nearly exclusively A site states, as expected for an inverse spinel ferrimagnetic state.

\section{Charge and orbital order}

The original model for charge order in magnetite ${ }^{2,35,50}$ set forward by Verwey had chains of octahedral Fe ions which were either $\mathrm{Fe}^{2+}$ or $\mathrm{Fe}^{3+}$. These chains are the $\mathrm{B} 1, \mathrm{~B} 2$, and mixed B3/B4 chains illustrated in Fig. 2. The structure of magnetite can be viewed as a lattice of corner sharing $\mathrm{Fe}_{4} \mathrm{O}_{4}$ cubes formed by perpendicular chains in adjacent vertical layers. The four Fe ions in each cube are arranged in a tet-

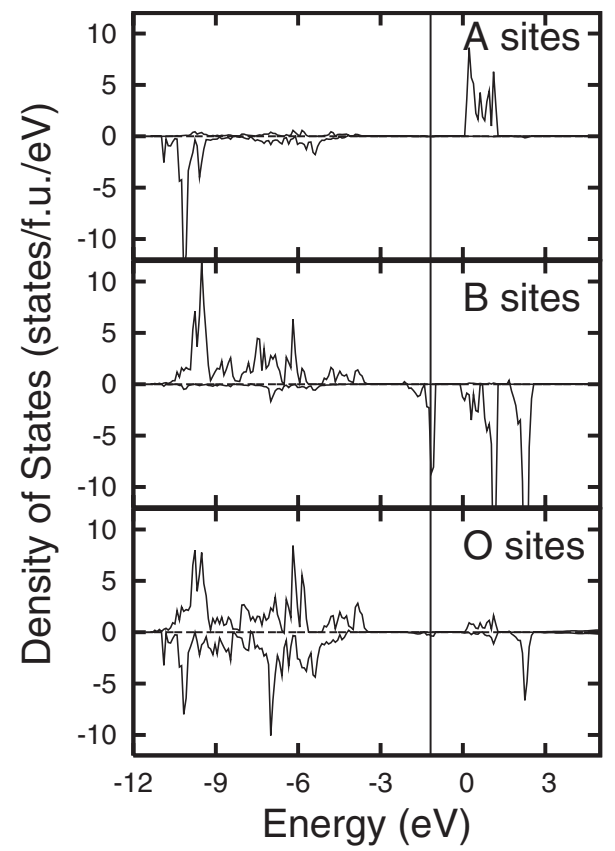

FIG. 5. Density of states for $\mathrm{Fe}_{3} \mathrm{O}_{4}$ in the $F d \overline{3} m$ unit cell from a DFT-GGA calculation projected onto tetrahedral and octahedral $\mathrm{Fe}$ and $\mathrm{O}$ sites. The Fermi energy is marked by a vertical line at $-1.2 \mathrm{eV}$.
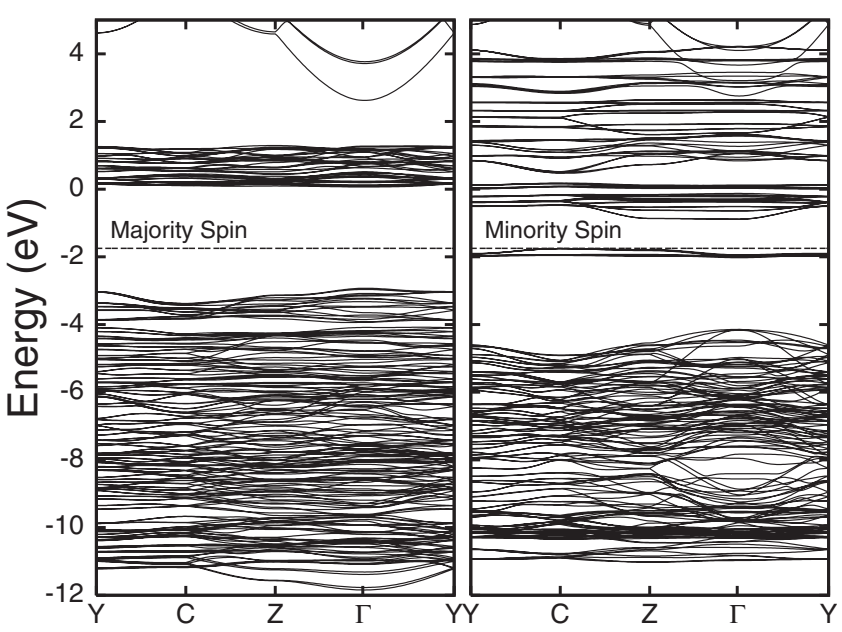

FIG. 6. Electronic band structure of $\mathrm{Fe}_{3} \mathrm{O}_{4}$ in the $P 2 / c$ unit cell. The valence band maximum is shown as a dashed line.

rahedron and there are two types of cube, containing B1a$\mathrm{B} 1 \mathrm{~b}-\mathrm{B} 3-\mathrm{B} 4$ and B2a-B2b-B3-B4 ions. On the grounds that electrostatic repulsions of ordered charges dominate the total energy in magnetite, Anderson ${ }^{36}$ argued that there should be two $\mathrm{Fe}^{2+}$ and two $\mathrm{Fe}^{3+}$ ions in each type of cube. Fe tetrahedra formed by intersecting chains in the Verwey model therefore had two ions of each valence and fulfilled the Anderson criterion $^{3}$ for minimal Coulomb repulsion.

These ideas have been contradicted recently by several DFT $+U$ calculations ${ }^{15,16,19}$ and conclusions of experimental

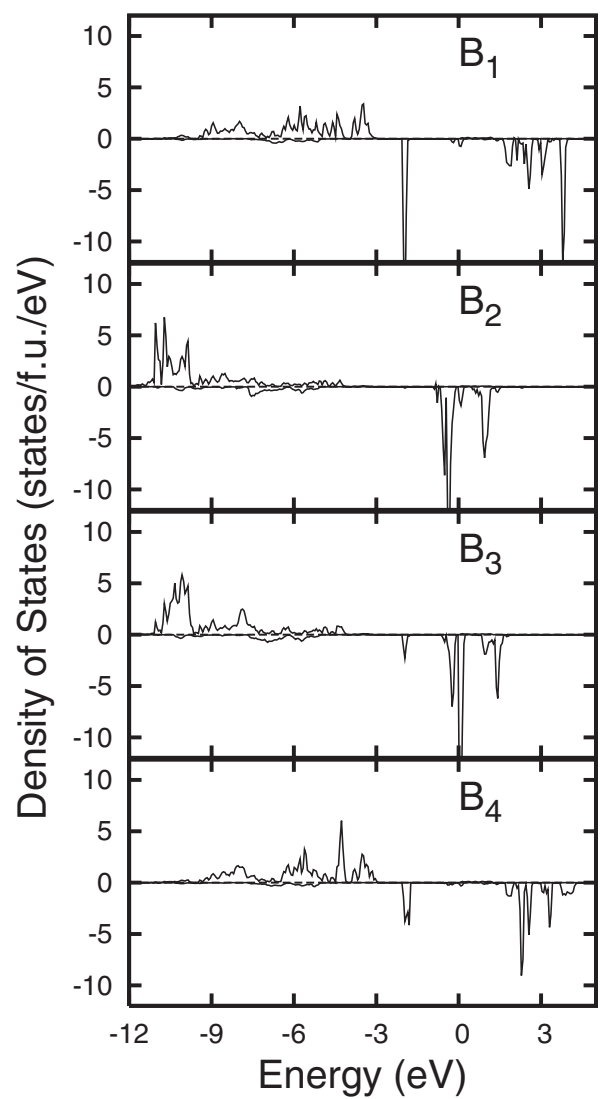

FIG. 7. Density of states for $\mathrm{Fe}_{3} \mathrm{O}_{4}$ in the $P 2 / c$ unit cell projected onto Fe B sites. 
TABLE III. Magnetite Mulliken net charge populations and magnetic moments on $\mathrm{Fe} \mathrm{A}$ and $\mathrm{B}$ sites.

\begin{tabular}{lcc}
\hline \hline Site & Net charge $e$ & Magnetic moment $\mu_{B}$ \\
\hline A1 & 2.10 & -4.15 \\
A2 & 2.10 & -4.15 \\
B1 & 1.83 & 3.76 \\
B2 & 2.17 & 4.26 \\
B3 & 2.11 & 4.18 \\
B4 & 1.81 & 3.73 \\
\hline \hline
\end{tabular}

work. ${ }^{8}$ Mulliken net charge populations and magnetic moments for magnetite with equilibrium atomic positions obtained with lattice parameters fixed at experimental values are given in Table III. Fe $3 d$ orbital populations from B3LYP functional calculations are compared to populations derived from several DFT $+U$ and a SIC-DFT calculation in Table IV.

Net charges on $\mathrm{B}$ site $\mathrm{Fe}$ ions range between 1.81e and $2.17 e$. $\mathrm{B} 1$ and $\mathrm{B} 4$ sites have charges of $1.81 e$ and $1.83 e$, respectively, and $\mathrm{B} 2$ and $\mathrm{B} 3$ sites have charges of 2.17e and $2.11 e$, respectively. Differences in net charges of $\mathrm{B} 1 \mathrm{a}$ and $\mathrm{B} 1 \mathrm{~b}$ and $\mathrm{B} 2 \mathrm{a}$ and $\mathrm{B} 2 \mathrm{~b}$ ions are less than 0.001e. Magnetic moments of B1 and B4 sites are $3.76 \mu_{B}$ and $3.73 \mu_{B}$ and moments of B2 and B3 sites are $4.26 \mu_{B}$ and $4.17 \mu_{B}$. The charge disproportionation parameters $\delta_{12}$ and $\delta_{34}$ introduced in Sec. I are 0.18 and 0.16 between B1/B2 and B3/B4 sites, somewhat larger than those derived from RXD, 0.12 and 0.10 , respectively. ${ }^{12}$ When the HF exchange weight parameter in Eq. (1) is reduced to 0.15 , these values change to 0.14 and 0.08 and straddle the experimentally derived values. Table IV shows that charge and spin disproportionation is explained by $\mathrm{Fe} 3 d$ orbital populations. B1 and B4 sites have $3 d$ populations of $6.10 e$ and $6.13 e$, close to the formal value of $6 e$ for an $\mathrm{Fe}^{2+}$ ion. $\mathrm{B} 2$ and $\mathrm{B} 3$ sites have $3 d$ populations of 5.66e and 5.71e, closer to formal populations of $\mathrm{Fe}^{2+}$ than $\mathrm{Fe}^{3+}$. Inspection of Table IV also shows that $\mathrm{Fe} 3 d$ populations predicted by the B3LYP functional lie within the range of $3 d$ populations obtained in three $\mathrm{DFT}+U$

TABLE IV. Comparison of $\mathrm{Fe}_{3} \mathrm{O}_{4}$ Mulliken charge populations on octahedral and tetrahedral $\mathrm{Fe} 3 d$ sites and band gaps in $\mathrm{eV}$ predicted by B3LYP, DFT $+U$, and SIC-DFT methods.

\begin{tabular}{lccccc}
\hline \hline Site & B3LYP & DFT $+U^{\mathrm{a}}$ & $\mathrm{DFT}+U^{\mathrm{b}}$ & $\mathrm{DFT}+U^{\mathrm{c}}$ & SIC-DFT $^{\mathrm{d}}$ \\
\hline A1 & 5.73 & & & 5.70 & 6.56 \\
$\mathrm{~A} 2$ & 5.73 & & & 5.70 & 6.56 \\
B1 & 6.10 & 5.57 & 6.04 & 5.95 & 7.74 \\
B2 & 5.66 & 5.41 & 5.73 & 5.66 & 7.45 \\
B3 & 5.71 & 5.44 & 5.92 & 5.72 & 7.15 \\
B4 & 6.13 & 5.58 & 6.03 & 5.92 & 7.35 \\
Band gap & 0.87 & 0.2 & 0.14 & 0.0 & 0.35 \\
\hline
\end{tabular}

\footnotetext{
${ }^{\text {aReference } 15 .}$

${ }^{\mathrm{b}}$ Reference 20 .

${ }^{\mathrm{C}}$ Reference 17

${ }^{\mathrm{d}}$ Reference 14 .
}
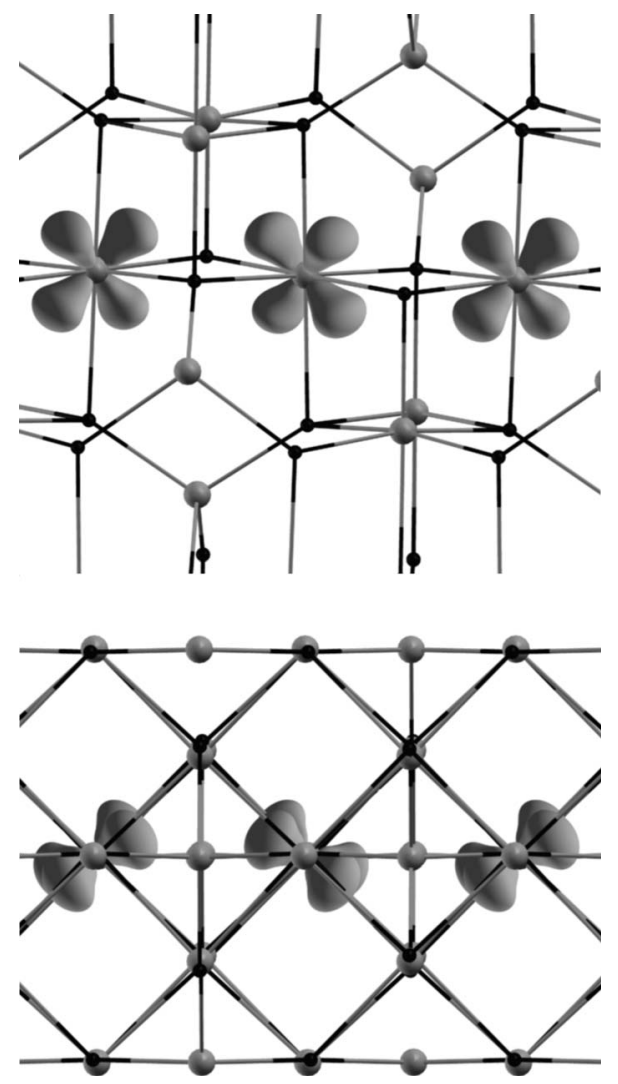

FIG. 8. Charge density difference plot for $\mathrm{Fe} \mathrm{B} 1$ sites of $\mathrm{Fe}_{3} \mathrm{O}_{4}$. (Top panel) View along $b$ crystallographic axis; (bottom panel) view along $c$ axis.

calculations ${ }^{15,17,20}$ and that $3 d$ populations predicted by a SIC-DFT calculation ${ }^{14}$ lie well outside this range. Band gaps predicted by $\mathrm{DFT}+U$ calculations range from 0.0 to $0.2 \mathrm{eV}$, the SIC-DFT band gap is $0.35 \mathrm{eV}$, and the B3LYP band gap is $0.87 \mathrm{eV}$. As noted earlier, the band gap predicted in hybrid functional calculations depends sensitively on the weight of HF exchange used. When the HF exchange weight parameter is reduced to $A=0.15$, the predicted band gap is reduced to $0.32 \mathrm{eV}$. Differences in single particle energy eigenvalues in DFT calculations cannot be interpreted as ionization potentials, strictly speaking. Disappearance of the gap, however, does indicate the onset of metallic behavior.

Charge order in magnetite is visualized in charge density difference plots in Figs. 8 and 9. Charge density difference plots are generated by subtracting a superposition of selfconsistent densities of $\mathrm{Fe}^{3+}$ and $\mathrm{O}^{2-}$ ions in vacuum from the self-consistent density for the $\mathrm{Fe}_{3} \mathrm{O}_{4}$ crystal. The difference function shows the distribution of the eight electrons per unit cell omitted from the reference density.

Figure 8 shows the charge density difference in the vicinities of the $\mathrm{B} 1 \mathrm{a}$ and $\mathrm{B} 1 \mathrm{~b}$ sites. The charge ordered electron is in a $t_{2 g}$ state and lies roughly within the four $\mathrm{O} 2$ and $\mathrm{O} 6$ ions illustrated in Fig. 3 (top panel). The presence of this orbital ordered electron explains the long $\mathrm{B} 1 \mathrm{Fe}-\mathrm{O} 2$ and $\mathrm{Fe}-\mathrm{O} 6$ bonds. A similar plot for the B2 site (not shown) shows no indication of extra charge on that site. Figure 9 shows a charge density difference plot for the plane containing the B3 and B4 sites. The B3 site shows only a diffuse spherical 

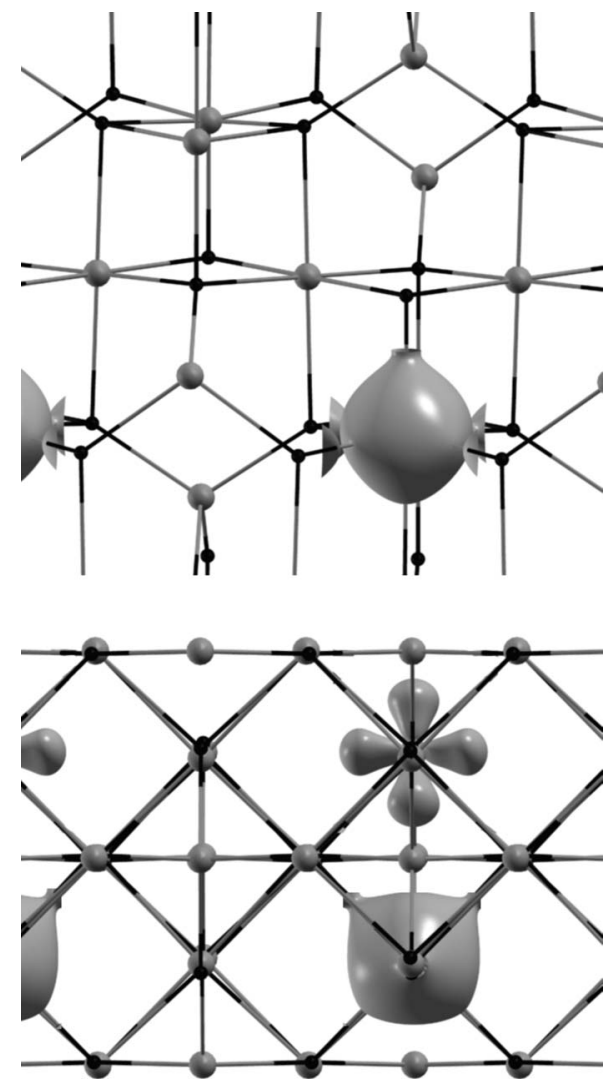

FIG. 9. Charge density difference plot for Fe B3 and B4 sites of $\mathrm{Fe}_{3} \mathrm{O}_{4}$. (Top panel) $\mathrm{B} 3$ sites viewed along crystallographic $a$ axis; (bottom panel) B3 and B4 sites viewed along $c$ axis.

isosurface and no charge localization, while the B4 site shows an electron in a $t_{2 g}$ orbital lying in the $a b$ plane of the $P 2 / c$ unit cell. The orbital ordered electron on the B4 site explains the long B4 Fe-O5 and Fe-O6 bonds (Fig. 3, bottom panel).

Patterns of bond lengths around the B1 site from x-ray data $^{8}$ and the B3LYP calculation are the same: bonds ranging in length from 2.120 to $2.131 \AA$ are predicted by the B3LYP calculation in the plane around B1 sites containing the $t_{2 g}$ electron. There are shorter bonds ranging from 2.045 to $2.046 \AA$ in the direction perpendicular to that plane. The ordered orbital on $\mathrm{B} 4$ sites lies parallel to the $a b$ crystallographic plane. $\mathrm{Fe}-\mathrm{O}$ bonds at $\mathrm{B} 4$ sites range between 2.095 and $2.117 \AA$ while there is one long Fe-O bond $(2.119 \AA)$ and one short bond $(2.043 \AA)$ perpendicular to the plane containing the orbital order. Bond lengths around B2 sites are nearly equivalent, ranging from 1.997 to $2.026 \AA$ in length. Bond lengths around B3 sites range from 1.981 to $2.108 \AA$ even though there is apparently no orbital order on that site (Fig. 9). A similar pattern of charge order has been found in DFT $+U$ calculations. ${ }^{15,16,19}$

\section{Lorentz oscillator model for IR conductivity}

In order to compare results of phonon calculations to phonons observed as peaks in IR conductivity measurements, ${ }^{42}$ the IR conductivity was calculated using a
TABLE V. Phonon frequencies in $F d \overline{3} m$ magnetite from experiment and DFT-GGA calculations in $\mathrm{cm}^{-1}$.

\begin{tabular}{lccccc}
\hline \hline DFT $^{\mathrm{a}}$ & DFT $^{\mathrm{b}}$ & DFT $^{\mathrm{c}}$ & Expt. $^{\mathrm{d}}$ & Irrep. & Activity \\
\hline 147 & 136 & 148 & & $T_{2 u}$ & \\
196 & 161 & 188 & 200 & $T_{1 u}$ & $\mathrm{IR}$ \\
204 & 170 & & & $E_{u}$ & \\
208 & 195 & 235 & 193 & $T_{2 g}$ & $\mathrm{R}$ \\
334 & 267 & & & $T_{1 g}$ & \\
343 & 309 & & & $T_{1 u}$ & $\mathrm{IR}$ \\
353 & 287 & 293 & & $A_{2 u}$ & \\
363 & 265 & & 308 & $E_{g}$ & $\mathrm{R}$ \\
389 & 323 & 327 & 350 & $T_{1 u}$ & $\mathrm{IR}$ \\
410 & 344 & & & $T_{2 u}$ & \\
465 & 400 & & 450 & $T_{2 g}$ & $\mathrm{R}$ \\
522 & 423 & & & $E_{u}$ & \\
562 & 538 & & 560 & $T_{1 u}$ & IR \\
565 & 525 & & 540 & $T_{2 g}$ & $\mathrm{R}$ \\
584 & 589 & & 670 & $A_{g}$ & $\mathrm{R}$ \\
657 & 598 & & & $A_{2 u}$ & \\
\hline \hline
\end{tabular}

aThis work.

${ }^{\mathrm{b}}$ Reference 22 .

${ }^{\mathrm{c}}$ Reference 46.

${ }^{\mathrm{d}}$ Reference 42.

LO model. The conductivity tensor $\sigma(\omega)$ is given by a sum over phonon modes,

$$
\begin{gathered}
\sigma\left(\omega / \mathrm{cm}^{-1}\right)=\frac{\omega}{60 \epsilon_{o} V} \sum_{i} \frac{Z_{i} Z_{i}^{T} \gamma \omega}{\left(\omega_{i}^{2}-\omega^{2}\right)^{2}+(\gamma \omega)^{2}}, \\
Z_{i}=\frac{z_{j} x_{i j}}{\sqrt{m_{j}}},
\end{gathered}
$$

where $\omega_{i}$ are phonon frequencies, $\gamma$ is a damping parameter, chosen to be $6 \mathrm{~cm}^{-1}, z_{j}$ are Born charges, $x_{i j}$ is the $j$ th component of the $i$ th phonon eigenvector, $m_{j}$ are atomic masses, and $V$ is the unit cell volume. It was not possible to obtain Born charges for $\mathrm{Fe}_{3} \mathrm{O}_{4}$ using the CRYSTAL package, so instead Born charges of $+2.67 e$ and $-2.00 e$ were used for all $\mathrm{Fe}$ and $\mathrm{O}$ ions, respectively.

\section{E. Phonons in the high temperature $F d \overline{3} m$ unit cell}

Symmetry analysis of the $F d \overline{3} m$ structure of magnetite shows that the 42 vibrational+translational modes consist of 12 Raman active modes $\left(A_{g}+E_{g}+3 T_{2 g}\right), 15$ IR active modes $\left(5 T_{2 u}\right), 3$ of which are translational modes, and 15 optically inactive modes $\left(2 B_{u}+2 E_{u}+T_{1 g}+2 T_{2 u}\right)$. Mode frequencies from experiment, the present work, and other DFT-GGA calculations are shown in Table V. Basis sets, $k$ point sampling conditions, etc., used in calculations reported in this work are described in the Appendix.

IR active modes are found in experiment at room temperature at 200, 350, and $560 \mathrm{~cm}^{-1}$; the mode around 


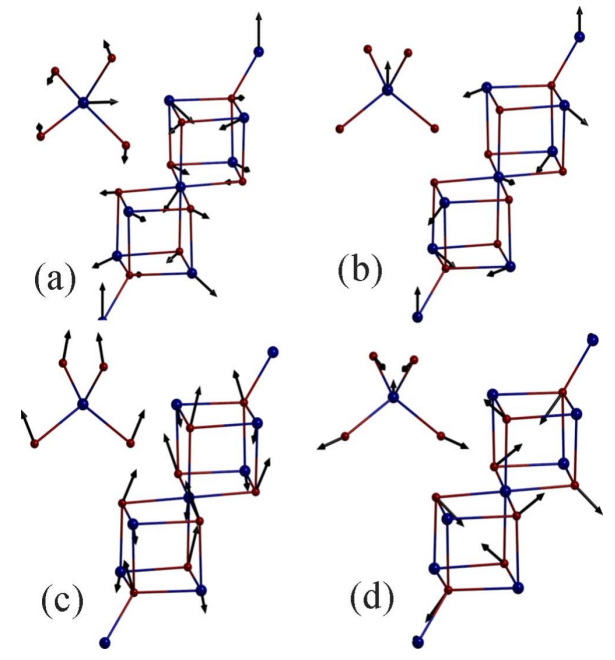

FIG. 10. (Color online) IR active phonon mode eigenvectors of $T_{1 u}$ symmetry from DFT-GGA calculation on $\mathrm{Fe}_{3} \mathrm{O}_{4}$ in the $F d \overline{3} m$ unit cell. Mode frequencies are (a) $196 \mathrm{~cm}^{-1}$, (b) $343 \mathrm{~cm}^{-1}$, (c) $389 \mathrm{~cm}^{-1}$, and (d) $562 \mathrm{~cm}^{-1}$. Displacements of $\mathrm{O}$ ions about tetrahedrally coordinated $\mathrm{Fe}^{3+}$ A site ions are shown separately for each mode.

$200 \mathrm{~cm}^{-1}$ is weak in the most recent data ${ }^{42}$ and is completely absent from the earlier data of Degiorgi et al. ${ }^{41}$ The modes at 350 and $560 \mathrm{~cm}^{-1}$ are the main features in the vibrational IR conductivity spectrum at room temperature. The three DFTGGA calculations in Table $\mathrm{V}$ predict the lowest $T_{1 u}$ mode frequency between 161 and $196 \mathrm{~cm}^{-1}$. Higher $T_{1 u}$ modes are predicted to lie between 309 and $343 \mathrm{~cm}^{-1}$, between 323 and $389 \mathrm{~cm}^{-1}$, and between 538 and $562 \mathrm{~cm}^{-1}$. The two highest frequency $T_{1 u}$ modes carry the great majority of the IR oscillator strength in an LO model of the IR conductivity. Frequencies for these two modes are predicted to be 389 and $562 \mathrm{~cm}^{-1}$ in this work; these compare well with experimental values of 350 and $560 \mathrm{~cm}^{-1}$. $^{42}$ The IR spectrum at room temperature therefore consists of the following: a $T_{1 u}$ mode at $200 \mathrm{~cm}^{-1}$ with little oscillator strength; a second $T_{1 u}$ mode, predicted to lie at $343 \mathrm{~cm}^{-1}$, is a weak shoulder in the strongest mode, which is observed at $350 \mathrm{~cm}^{-1}$ and predicted at $389 \mathrm{~cm}^{-1}$; and the highest $T_{1 u}$ mode is at $560 \mathrm{~cm}^{-1}$, in good agreement with the predicted value of $562 \mathrm{~cm}^{-1}$. Ion displacements in IR active phonons in $F d \overline{3} \mathrm{~m}$ $\mathrm{Fe}_{3} \mathrm{O}_{4}$ are illustrated in Fig. 10 .

Raman active modes are observed in experiment at room temperature at 193, 308, 540, and $670 \mathrm{~cm}^{-1}$ by Gasparov et $a l^{42}$ and at 193, 306, 538, and $668 \mathrm{~cm}^{-1}$ by Shebanova and Lazor. ${ }^{44}$ There is therefore good agreement between these recent studies. Shebanova and Lazor ${ }^{44}$ assigned the modes at 193 and $538 \mathrm{~cm}^{-1}$ to $T_{2 g}$ modes and the modes at 306 and $668 \mathrm{~cm}^{-1}$ to $E_{g}$ and $A_{g}$ modes, respectively. They assigned a weak feature observed at $490 \mathrm{~cm}^{-1}$ in another study ${ }^{51}$ to the third $T_{2 g}$ mode. There is a weak peak at $450 \mathrm{~cm}^{-1}$ in the room temperature data in Ref. 42 . The ranges of positions of Raman active modes predicted by the DFT calculations in Table V are 195-235, 400-465, and 525-565 $\mathrm{cm}^{-1}$ for $T_{2 g}$ modes, $265-363 \mathrm{~cm}^{-1}$ for the $E_{g}$ mode, and $584-589 \mathrm{~cm}^{-1}$ for the $A_{g}$ mode. Eigenvectors for Raman active modes in

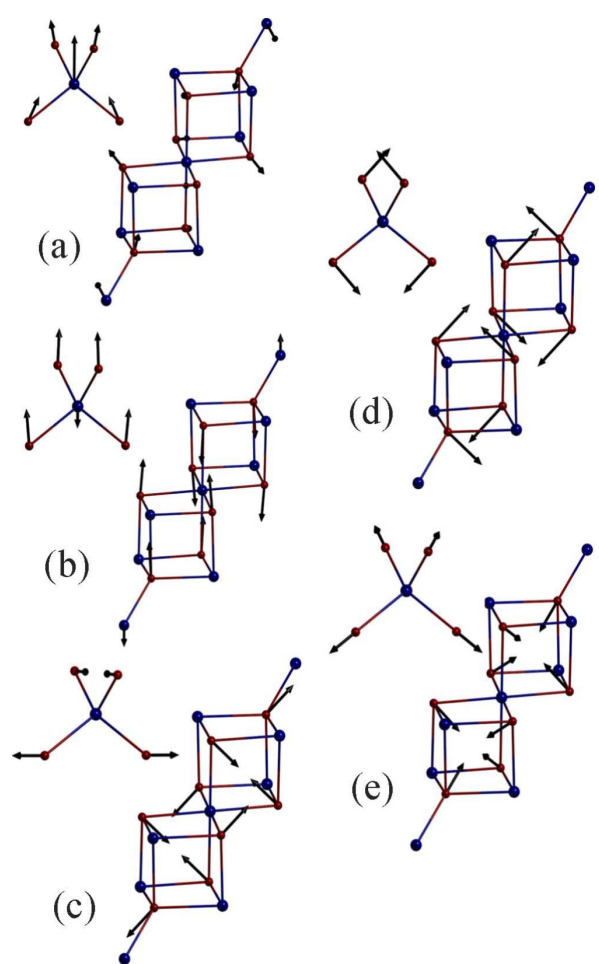

FIG. 11. (Color online) Raman active phonon mode eigenvectors from DFT-GGA calculations on $\mathrm{Fe}_{3} \mathrm{O}_{4}$ in the $F d \overline{3} m$ unit cell. Mode frequencies and symmetries are (a) $223 \mathrm{~cm}^{-1} T_{2 g}$, (b) $465 \mathrm{~cm}^{-1} T_{2 g}$, (c) $565 \mathrm{~cm}^{-1} T_{2 g}$, (d) $363 \mathrm{~cm}^{-1} E_{g}$, and (e) $584 \mathrm{~cm}^{-1} A_{g}$. Displacements of $\mathrm{O}$ ions about tetrahedrally coordinated $\mathrm{Fe}^{3+} \mathrm{A}$ site ions are shown separately for each mode.

$F d \overline{3} m \mathrm{Fe}_{3} \mathrm{O}_{4}$ from the GGA-DFT calculation are shown in Fig. 11. The assignment by Shebanova and Lazor ${ }^{44}$ is therefore in agreement with the mode frequencies from these DFT calculations.

\section{F. Phonons in the low temperature $P 2 / c$ unit cell}

There are 56 ions in the $P 2 / c$ unit cell of $\mathrm{Fe}_{3} \mathrm{O}_{4}$; the 168 vibrational+translational modes at the $\Gamma$ point of the Brillouin zone consist of $38 A_{g}+40 B_{g}+44 A_{u}+46 B_{u}$ modes. Symmetry operations for the $P 2 / c$ space group with a unique $b$ axis are $E, C_{2}(y)+0.5 z, i$, and $\sigma_{h}+0.5 z . A(B)$ modes are even (odd) with respect to the $C_{2}$ rotation, $g(u)$ modes are even (odd) with respect to inversion, the $A_{g}$ and $B_{u}$ modes are even with respect to the mirror plane, and the $A_{u}$ and $B_{g}$ modes are odd. Figure 2 shows that B1a Fe ions lie on the $C_{2}$ axis and $\mathrm{B} 2 \mathrm{Fe}$ ions almost lie in the mirror plane. $A_{u}$ and $B_{u}$ modes are IR active and couple to electric fields polarized along the $y$ and $x, z$ axes, respectively, when the unique axis is $b$ and $x, y$, and $z$ axes are parallel or almost parallel to the $a, b$, and $c$ axes. $A_{g}$ and $B_{g}$ modes are Raman active. $A_{g}$ modes are detected in an $X X$ experimental geometry ${ }^{44}$ in which incident and scattered radiations have the same polarization, while $B_{g}$ modes are detected in an $X Y$ geometry where polarizations of incident and scattered radiations are at right angles.

Experimental IR conductivity data for $\mathrm{Fe}_{3} \mathrm{O}_{4}$ at $40 \mathrm{~K}$ from Ref. 42 are reproduced in Fig. 12 and show two strong bands 


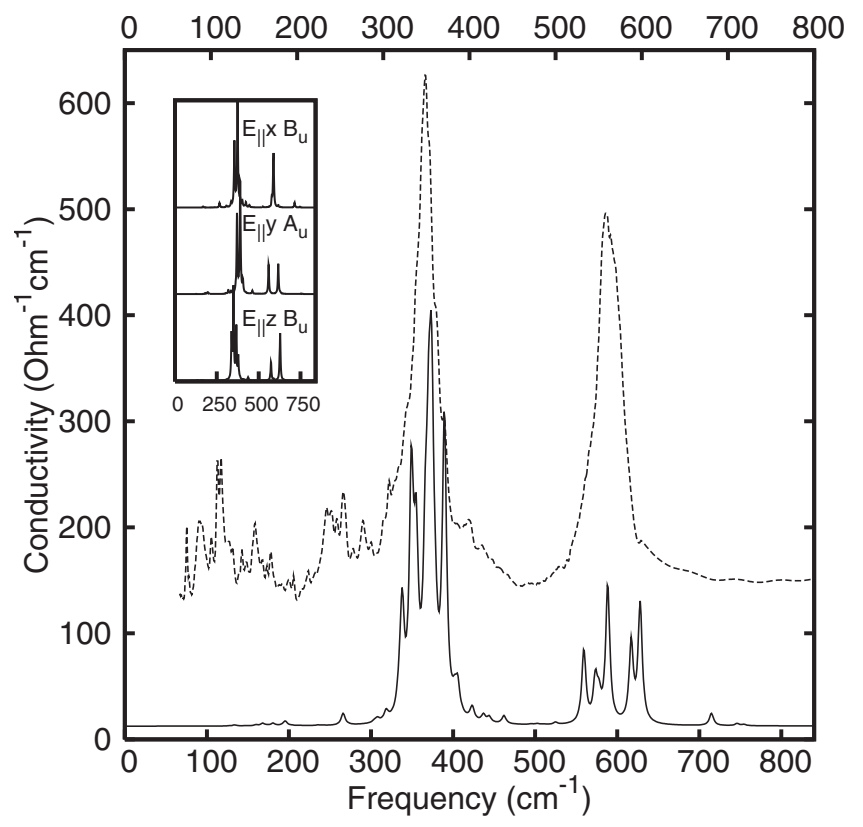

FIG. 12. IR conductivity of $\mathrm{Fe}_{3} \mathrm{O}_{4}$ at $40 \mathrm{~K}$ reproduced from Ref. 42 (dotted curve) and from an $\mathrm{LO}$ model using phonon modes from B3LYP calculation on $\mathrm{Fe}_{3} \mathrm{O}_{4}$ in the $P 2 / c$ unit cell (solid curve). The orientationally averaged spectrum from the LO model is drawn with a scale which differs from that for experiment by $5 \%$, as explained in the text. Inset shows the decomposition of the averaged IR conductivity into components excited by electric field vectors aligned with Cartesian $x, y$, and $z$ axes.

between 300 and $400 \mathrm{~cm}^{-1}$ and between 550 and $650 \mathrm{~cm}^{-1}$. There are also bands around 100 and $250 \mathrm{~cm}^{-1}$. The LO model in Eq. (2) was used to calculate the conductivity of $\mathrm{Fe}_{3} \mathrm{O}_{4}$ using $A_{u}$ and $B_{u}$ phonon eigenmodes. Vibrational frequencies (and other properties such as band gap ${ }^{30}$ ) predicted by hybrid DFT methods depend quite sensitively on the weight of $\mathrm{HF}$ exchange used in calculations; in the case of $\mathrm{Fe}_{3} \mathrm{O}_{4}$, increasing the weight from $20 \%$ (B3LYP) to $30 \%$ increases mode frequencies in $\mathrm{Fe}_{3} \mathrm{O}_{4}$ by $10 \%$. When the experimental data and the results of the LO model are compared, it is clear that peaks in the IR conductivity from the LO model are too high in frequency by about $5 \%$. The results of the LO model and the experimental data are plotted in Fig. 12 with scales that differ by $5 \%$ so that the scaled LO model curve can be directly compared to experiment. The main LO model curve in Fig. 12 shows orientationally averaged conductivities for electric fields applied along three Cartesian directions. The inset of Fig. 12 shows LO model conductivities for each applied field direction. Inspection of the inset shows that the modes in the higher frequency IR band occur as split pairs for fields along the $x$ and $y$ ( $a$ and $b$ crystallographic) directions. This splitting arises because of charge order on octahedral B sites.

The IR active $T_{1 u}$ mode predicted by DFT-GGA at $389 \mathrm{~cm}^{-1}$ in the $F d \overline{3} m$ unit cell and observed in experiment around $350 \mathrm{~cm}^{-1}$ (Table V) is replaced by $10 A_{u}$ and $12 B_{u}$ modes in the range of $300-400 \mathrm{~cm}^{-1}$ in the B3LYP calculation. The mode predicted by DFT-GGA at $562 \mathrm{~cm}^{-1}$ in the $F d \overline{3} m$ unit cell and observed in experiment around $560 \mathrm{~cm}^{-1}$
TABLE VI. Strongly IR active phonon modes in $P 2 / c$ magnetite from B3LYP calculations. Ion sites on which phonon eigenvector amplitudes are primarily concentrated and mode frequencies in $\mathrm{cm}^{-1}$ are given.

\begin{tabular}{ccc}
\hline \hline Freq. & Irrep. & Eigenvector \\
\hline 370 & $A_{u}$ & $\mathrm{~B} 4 \mathrm{O} 4 \mathrm{O} 5 \mathrm{O} 6$ \\
389 & $A_{u}$ & $\mathrm{~B} 2 \mathrm{~B} 3 \mathrm{O} 1 \mathrm{O} 3 \mathrm{O} 5 \mathrm{O} 6$ \\
349 & $B_{u}$ & $\mathrm{~B} 2 \mathrm{O} 4$ \\
373 & $B_{u}$ & $\mathrm{~B} 2 \mathrm{~B} 3 \mathrm{O} 1 \mathrm{O} 4 \mathrm{O} 5 \mathrm{O} 6$ \\
559 & $A_{u}$ & O3 O4 \\
617 & $A_{u}$ & O1 O2 \\
573 & $B_{u}$ & O5 \\
627 & $B_{u}$ & $\mathrm{~B} 2$ O6 \\
\hline \hline
\end{tabular}

(Table VI) is replaced by $5 A_{u}$ and $5 B_{u}$ modes in the range of $550-650 \mathrm{~cm}^{-1}$ in the B3LYP calculation. Mode frequencies and concentration of eigenvector amplitudes on specific sites in the $P 2 / c$ unit cell, for the four most optically active $A_{u}$ and $B_{u}$ modes in each band, are given in Table VI. Modes with greatest IR activity in the band between 300 and $400 \mathrm{~cm}^{-1}$ are found at 370 and $389 \mathrm{~cm}^{-1}\left(A_{u}\right)$ and at 349 and $373 \mathrm{~cm}^{-1}\left(B_{u}\right)$. Modes with greatest IR activity in the band between 550 and $650 \mathrm{~cm}^{-1}$ are found at 559 and $617 \mathrm{~cm}^{-1}\left(A_{u}\right)$ and at 573 and $627 \mathrm{~cm}^{-1}\left(B_{u}\right)$ (Fig. 12). Ion displacements in IR active phonons with the largest optical activity in the band between 300 and $400 \mathrm{~cm}^{-1}$ are available in Ref. 52.

Inspection of the $A_{u}$ mode eigenvectors at 370 and $389 \mathrm{~cm}^{-1}$ shows that ion displacements in these modes are concentrated on specific $B$ site chains. The mode at $370 \mathrm{~cm}^{-1}$ is concentrated on $\mathrm{B} 4, \mathrm{O} 5$, and $\mathrm{O} 6$ sites of the B3/B4 chain and especially on $\mathrm{O} 4$ sites in the B2 chain (Table VI). The mode at $389 \mathrm{~cm}^{-1}$ is concentrated on B3, $\mathrm{O} 5$, and $\mathrm{O} 6$ sites in the B3/B4 chain and also on the B2 and $\mathrm{O} 3$ sites in the $\mathrm{B} 2$ chain. These modes are similar in character but are concentrated either on $\mathrm{O} 3$ or $\mathrm{O} 4$ ions in the $\mathrm{B} 2$ chain. The $349 \mathrm{~cm}^{-1} B_{u}$ mode eigenvector is strongly concentrated on $\mathrm{B} 2$ and $\mathrm{O} 4$ sites in the $\mathrm{B} 2$ chains and the $374 \mathrm{~cm}^{-1}$ mode eigenvector is spread over all B site layers. Charge order must induce changes in force constants for interactions between $\mathrm{Fe}$ ions and their $\mathrm{O}$ neighbors. Symmetry breaking in magnitudes of force constants on going from the cubic $F d \overline{3} m$ unit cell to the $P 2 / c$ monoclinic cell will result in ion displacements in specific modes becoming concentrated on specific ions and splitting of frequencies of phonons which are degenerate in the cubic unit cell. The extent of concentration of modes on specific $\mathrm{O}$ ions is much greater for the higher frequency IR and Raman active bands which predominantly involve $\mathrm{O}$ ion displacements.

The IR conductivity band between 550 and $650 \mathrm{~cm}^{-1}$ is generated by modes which are essentially completely concentrated on $\mathrm{O}$ ions. They consist of out-of-phase A site $\mathrm{Fe}-\mathrm{O}$ stretching in both the $F d \overline{3} m$ and $P 2 / c$ unit cells. In the $P 2 / c$ unit cell, modes are concentrated on $\mathrm{O}$ ions in particular $\mathrm{B}$ site chains, whereas in the $F d \overline{3} m$ unit cell, symmetry dictates that displacement amplitudes on each $\mathrm{O}$ ion are equal in 
magnitude. The $559 \mathrm{~cm}^{-1} A_{u}$ mode in Fig. 13(a) is concentrated on $\mathrm{O} 3$ and $\mathrm{O} 4$ ions in $\mathrm{B} 2$ chains, the $617 \mathrm{~cm}^{-1} A_{u}$ mode is concentrated on $\mathrm{O} 1$ and $\mathrm{O} 2$ ions in $\mathrm{B} 1$ chains, the $573 \mathrm{~cm}^{-1} B_{u}$ mode is concentrated on $\mathrm{O} 5$ ions, and the $627 \mathrm{~cm}^{-1} B_{u}$ mode is concentrated on $\mathrm{B} 2$ and $\mathrm{O} 6$ ions.

The pattern of concentration of ion displacements on specific sites can be explained by charge order on B sites. The charge order described in Sec. II C shows that Fe ions in B2 chains are formally $\mathrm{Fe}^{3+}$ while $\mathrm{Fe}$ ions in $\mathrm{B} 1$ chains are formally $\mathrm{Fe}^{2+} . B_{u}$ modes in Figs. 13(c) and 13(d) are concentrated on B3/B4 chains but are split between $\mathrm{O} 5$ and $\mathrm{O} 6$ sites. O5 ions are bonded to $\mathrm{B} 2$ sites $\left(\mathrm{Fe}^{3+}\right)$ in the chain above or below, while $\mathrm{O} 6$ ions are bonded to $\mathrm{B} 1$ sites $\left(\mathrm{Fe}^{2+}\right)$ above or below. This difference in environment is sufficient to split the mode frequencies by $54 \mathrm{~cm}^{-1}$. Obviously, the greater the extent of charge ordering, the larger the splitting will be.

Since single modes in the $F d \overline{3} m$ phase are replaced by multiple modes in the $P 2 / c$ phase, broadening of IR band linewidths in experiment might be expected on cooling through $T_{V}$. Linewidths and frequency shifts in IR and Raman active modes which accompany the Verwey transition were reported previously. ${ }^{42}$ The $300-400 \mathrm{~cm}^{-1}$ band linewidth increases from 24 to $39 \mathrm{~cm}^{-1}$ and the $550-650 \mathrm{~cm}^{-1}$ band linewidth increases from 26 to $36 \mathrm{~cm}^{-1}$ on cooling through $T_{V}$. The experimental linewidth is too broad to distinguish individual modes in the $P 2 / c$ phase, but the $300-400 \mathrm{~cm}^{-1}$ band is certainly more asymmetric below $T_{V}$. However, the linewidth of the strong Raman line around $670 \mathrm{~cm}^{-1}$ actually decreases from 30 to $20 \mathrm{~cm}^{-1}$ on cooling through $T_{V \cdot}{ }^{42}$

The Raman spectrum for $\mathrm{Fe}_{3} \mathrm{O}_{4}$ at $5 \mathrm{~K}$ is shown in Fig. 14 for both $X X$ and $X Y$ experimental geometries. Changes in the Raman spectrum in the $X X$ experimental geometry on warming to $300 \mathrm{~K}$ are shown in the inset of Fig. 14. There are dramatic changes in the Raman spectrum in the $X X$ experimental geometry on cooling below $T_{V}$, in particular a mode appears around $165 \mathrm{~cm}^{-1}$, a broad band of modes appears between 200 and $500 \mathrm{~cm}^{-1}$, and a shoulder to the $A_{g}$ mode at $670 \mathrm{~cm}^{-1}$ appears around $650 \mathrm{~cm}^{-1}$. It is not possible to calculate Raman cross sections using the CRYSTAL package. Instead the $A_{g}$ and $B_{g}$ densities of modes at the $\Gamma$ point are compared to experimental spectra by representing each mode as a Lorentzian with a full width at half maximum (FWHM) of $6 \mathrm{~cm}^{-1}$. Frequency axis scales which differ by $5 \%$ for B3LYP calculation and experimental mode frequencies are used in the same manner as for the IR spectrum in Fig. 12. There are less dramatic changes in the $X Y$ geometry experimental spectrum (Fig. 14) on cooling through $T_{V}$. There is reasonable agreement between the density of modes from the B3LYP calculation and the positions of experimental lines in the $X X$ and $X Y$ geometries, except below $250 \mathrm{~cm}^{-1}$ in the $X Y$ geometry.

The most intense Raman lines in the room temperature spectrum of $\mathrm{Fe}_{3} \mathrm{O}_{4}$ are the $T_{2 g}$ and $A_{g}$ modes predicted by the DFT-GGA calculation in this work to lie at 565 and $584 \mathrm{~cm}^{-1}$, respectively. Experimental lines are observed at 540 and $670 \mathrm{~cm}^{-1}$. The B3LYP calculation on $\mathrm{Fe}_{3} \mathrm{O}_{4}$ in the $P 2 / c$ unit cell predicts $4 A_{g}$ and $4 B_{g}$ modes between 560 and
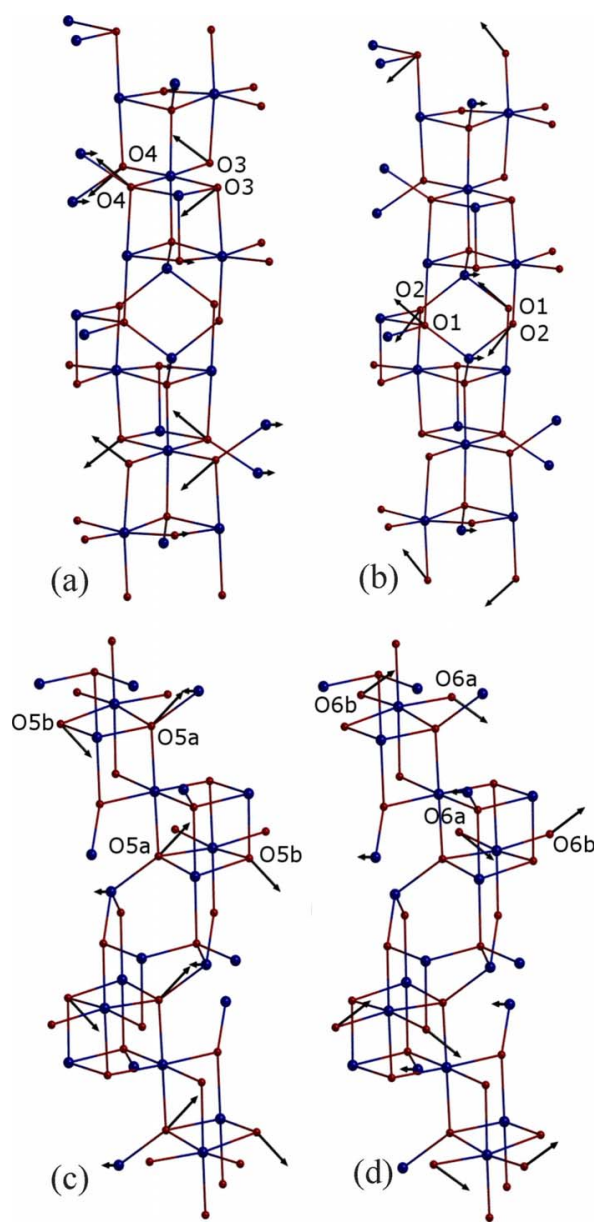

FIG. 13. (Color online) Phonon eigenvectors with largest optical activity in the band between 550 and $650 \mathrm{~cm}^{-1}$ from B3LYP calculation on $P 2 / c$ unit cell. Mode frequencies and irreducible representations are (a) $559 \mathrm{~cm}^{-1} A_{u}$, (b) $617 \mathrm{~cm}^{-1} A_{u}$, (c) $573 \mathrm{~cm}^{-1} B_{u}$, and (d) $627 \mathrm{~cm}^{-1} B_{u}$.

$640 \mathrm{~cm}^{-1}$ and $4 A_{g}$ and $4 B_{g}$ modes between 650 and $800 \mathrm{~cm}^{-1}$. These groups of modes replace the highest frequency $T_{2 g}$ and $A_{g}$ modes observed above $T_{V}$.

Raman active modes between 560 and $640 \mathrm{~cm}^{-1}$ differ in character from the $565 \mathrm{~cm}^{-1} T_{2 g}$ modes in $F d \overline{3} m \mathrm{Fe}_{3} \mathrm{O}_{4}$ (Fig. 11). The $8 A_{g}$ and $B_{g}$ modes between 560 and $640 \mathrm{~cm}^{-1}$ consist of out-of-phase breathing motions along A site $\mathrm{Fe}-\mathrm{O}$ bonds, similar to the $A_{u}$ and $B_{u}$ IR active modes shown in Fig. 13, but are even rather than odd with respect to inversion. Ion displacements for the four $B_{g}$ modes between 560 and $640 \mathrm{~cm}^{-1}$ are available in Ref. 52 . The pattern of concentration of eigenvector amplitude for the $B_{g}$ modes at 574 and 631 is the same as for the $A_{u}$ and $B_{u}$ IR active modes between 559 and $627 \mathrm{~cm}^{-1}$ (Table VI). Ion displacements are concentrated on $\mathrm{O} 3+\mathrm{O} 4$ ions in $\mathrm{B} 2$ chains, $\mathrm{O} 5$ ions in $\mathrm{B} 3 / \mathrm{B} 4$ chains, $\mathrm{O} 1+\mathrm{O} 2$ ions in $\mathrm{B} 1$ chains, and $\mathrm{O} 6$ ions in B3/B4 chains.

The $A_{g}$ mode at $584 \mathrm{~cm}^{-1}$ in the DFT-GGA calculation on $\mathrm{Fe}_{3} \mathrm{O}_{4}$ in the $F d \overline{3} m$ unit cell consists of breathing motions of A site Fe-O bonds [Fig. 11(e)]. The in-phase breathing motion induces large electronic polarizability changes with changes in phonon coordinate and hence this is the mode 


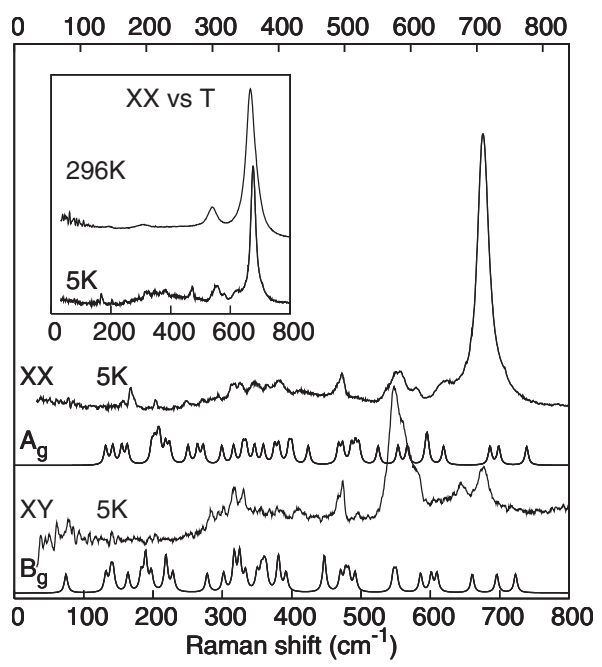

FIG. 14. Raman spectrum of $\mathrm{Fe}_{3} \mathrm{O}_{4}$ at $5 \mathrm{~K}$ and $A_{g}$ and $B_{g}$ mode densities from B3LYP calculation on $\mathrm{Fe}_{3} \mathrm{O}_{4}$ in the $P 2 / c$ unit cell. Experimental spectra measured in $X X$ and $X Y$ geometries are reproduced from Ref. 42. Mode frequencies from the LO model are drawn with a scale which differs from that for experiment by $5 \%$ as explained in the text. Inset shows the variation in the Raman spectrum with temperature below and above $T_{V}$

with the greatest Raman cross section. The $8 A_{g}$ and $B_{g}$ modes in the $P 2 / c$ unit cell between 650 and $800 \mathrm{~cm}^{-1}$ also consist of breathing motions of A site Fe-O bonds. Mode frequencies and concentration of ion displacements on specific $\mathrm{O}$ ions are given in Table VII. Eigenvectors corresponding to the $B_{g}$ mode at $693 \mathrm{~cm}^{-1}$ and the $A_{g}$ mode at $720 \mathrm{~cm}^{-1}$ are shown in Fig. 15. They have a breathing char-

TABLE VII. Raman active $A_{g}$ and $B_{g}$ phonon modes between 560 and $640 \mathrm{~cm}^{-1}$ and between 650 and $800 \mathrm{~cm}^{-1}$ from B3LYP calculation on $\mathrm{Fe}_{3} \mathrm{O}_{4}$ in the $P 2 / c$ unit cell. Ion sites on which phonon amplitudes are primarily concentrated and mode frequencies in $\mathrm{cm}^{-1}$ are given.

\begin{tabular}{ccc}
\hline \hline Freq. & Irrep. & Eigenvector \\
\hline 581 & $A_{g}$ & O2 O3 \\
595 & $A_{g}$ & O1 O2 \\
624 & $A_{g}$ & O5 O6 \\
626 & $A_{g}$ & O2 O3 O4 \\
574 & $B_{g}$ & O3 O4 \\
578 & $B_{g}$ & O5 \\
615 & $B_{g}$ & O1 O2 \\
631 & $B_{g}$ & O6 \\
650 & $A_{g}$ & O2 O5b \\
720 & $A_{g}$ & O5 O6 \\
733 & $A_{g}$ & O3 O4 \\
775 & $A_{g}$ & O1 O2 O6 \\
639 & $B_{g}$ & O2 O5 \\
693 & $B_{g}$ & O3 O4 \\
731 & $B_{g}$ & O2 O5 \\
759 & $B_{g}$ & O1 O2 O6 \\
\hline \hline
\end{tabular}
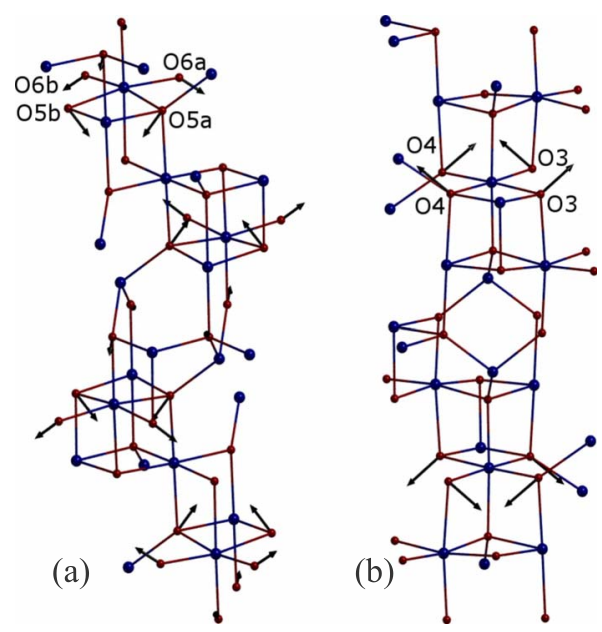

FIG. 15. (Color online) Raman active phonon eigenvectors from B3LYP calculation on $\mathrm{Fe}_{3} \mathrm{O}_{4}$ in the $P 2 / c$ unit cell between 650 and $800 \mathrm{~cm}^{-1}$. (a) $720 \mathrm{~cm}^{-1}\left(A_{g}\right)$; (b) $693 \mathrm{~cm}^{-1}\left(B_{g}\right)$.

acter about $\mathrm{A}$ site $\mathrm{Fe}^{3+}$ sites but concentration of the modes on particular O sites results in a "half-breathing" mode.

\section{DISCUSSION}

\section{A. Crystal structure}

The charge ordered state of magnetite below $T_{V}$ is characterized by distortions of $\mathrm{Fe} \mathrm{B}$ site octahedra, ${ }^{8}$ disproportionation of charge, ${ }^{8,9,12}$ and magnetic moments between B site octahedra and opening of a $d-d$ optical band gap. ${ }^{53}$ Octahedra surrounding $\mathrm{B} 1$ and $\mathrm{B} 4$ sites are expanded relative to $\mathrm{B} 2$ and B3 sites with mean $\mathrm{Fe}-\mathrm{O}$ bond lengths of $2.072 \AA$ (2.099) for the B1 site from experiment ${ }^{8}$ (or B3LYP energy minimization), $2.069 \AA$ (2.098) for the $\mathrm{B} 4$ site, $2.043 \AA$ (2.016) for the $\mathrm{B} 2$ site, and $2.050 \AA$ (2.027) for the $\mathrm{B} 3$ site. Similar computed mean $\mathrm{Fe}-\mathrm{O}$ bond lengths were obtained in a GGA $+U$ energy minimization calculation by Pinto and Elliott. ${ }^{19}$ Mean bond lengths obtained were $2.111 \AA$ A (B1), $2.108 \AA(\mathrm{B} 4), 2.033 \AA$ (B2), and $2.045 \AA$ (B3). Both energy minimization calculations predict $\mathrm{B} 1$ and $\mathrm{B} 4$ mean bond lengths to be greater than experiment by $0.03-0.04 \AA$ and they predict $\mathrm{B} 2$ and $\mathrm{B} 3$ mean bond lengths to be shorter than experiment by $0.01-0.03 \AA$. As noted in Sec. II C, the pattern of Jahn-Teller distortions about Fe B sites can be understood in terms of the particular pattern of orbital order found on those sites.

\section{B. Charge order}

BVSs calculated from alternation in mean $\mathrm{Fe}-\mathrm{O}$ bond length in $\mathrm{B}$ site octahedra have been interpreted as experimental evidence for charge order in magnetite ${ }^{8} \mathrm{Fe} \mathrm{B} 1$ and B4 sites were found to have a valence of +2.4 while B2 and B3 sites have a valence of +2.6 . Differences in net electronic charges on these sites have been calculated in DFT $+U$, SICDFT, and B3LYP functional calculations in order to obtain a theoretical estimate of the degree of charge disproportionation and charge order in magnetite. Here the charge disproportionation parameters were found to be $\delta_{12}=0.18$ for B1 
and B2 sites and $\delta_{34}=0.16$ for B3 and B4 sites. Jeng et al. ${ }^{18}$ investigated the dependence of charge disproportionation on the Hubbard $U$ parameter in their DFT $+U$ calculations. They found a charge disproportionation of $0.11 e$ and zero electronic band gap for a $U$ value of $4.0 \mathrm{eV}$, which increased to $0.19 e$ and a band gap of $0.63 \mathrm{eV}$ when $U$ was increased to $5.5 \mathrm{eV}$. The optimal value of $U$ was found to be $4.5 \mathrm{eV}$, which yielded a charge disproportionation of $0.15 e$ and band gap of $0.20 \mathrm{eV}$. These calculations were performed using the crystal structure of Wright et al.; ${ }^{8}$ when the crystal structure was allowed to relax the charge disproportionation remained fixed at $0.15 e$ and the band gap increased to $0.44 \mathrm{eV} .{ }^{18} \mathrm{Using}$ a $U$ value of $6.21 \mathrm{eV},{ }^{17}$ Madsen and Novak ${ }^{17}$ found a charge disproportionation of $0.24 e$ and Leonov et $a l .^{20}$ found a charge disproportionation of $0.24 e$ and a band gap of 0.18 $\mathrm{eV}$ using a $U$ value of $5.0 \mathrm{eV}$. Szotek et al. ${ }^{14}$ found a charge disproportionation of $0.36 e$ and a band gap of $0.35 \mathrm{eV}$ for the SIC-DFT ground state in the $P 2 / c$ unit cell. The measures of charge disproportionation used in calculations and experiment differ considerably; estimates by experimentalists were based on crystal structure and bond valence sums while estimates by theoreticians are based on projections of charge onto $\mathrm{Fe} \mathrm{B}$ sites. Charge disproportionation estimates from $\mathrm{DFT}+U$ calculations ${ }^{17,18,20}$ were based on total charges within spheres of limited radii centered on Fe B sites, while the B3LYP calculations reported here used a Mulliken population analysis. Nevertheless, all estimates show that the extent of charge disproportionation is apparently much less than complete disproportionation into $\mathrm{Fe}^{2+}$ and $\mathrm{Fe}^{3+}$ ions.

DFT $+U$ studies which reported orbital ordering, ${ }^{11,15,19,20}$ as well as the B3LYP calculations reported here, all yield essentially the same electronic band structure and charge and orbital ordered state for $\mathrm{Fe}_{3} \mathrm{O}_{4}$ in the $P 2 / c$ unit cell. The eight conduction electron bands around $-2 \mathrm{eV}$ in the right panel of Fig. 6 are localized on B1 and B4 sites. Density of states contributions to those bands from $\mathrm{O}$ or other $\mathrm{Fe} \mathrm{A}$ or $\mathrm{B}$ sites are small. The orbital order of this state, shown in Figs. 8 and 9, contains ordered $t_{2 g}$ electrons on B1 and B4 sites; the spherical density found on the B3 site (Fig. 9) is a screening charge from deeper in the valence band. These charges maintain a degree of charge neutrality over the B sites and reduce the charge disproportionation to the low observed values of $\sim 0.1$.

The $P 2 / c$ unit cell used here and in most recent computational work is not the full unit cell for $\mathrm{Fe}_{3} \mathrm{O}_{4}$. One study ${ }^{18}$ has considered charge order in the full $C c$ space group and finds that when this structure is relaxed, charge and orbital order along B1 chains is the same as in the $P 2 / c$ unit cell, but an alternating occupied/unoccupied order develops on B2 and B3/B4 chains. The well known Anderson criterion ${ }^{36}$ for minimal Coulomb repulsion in the magnetite structure requires that each cube contains two $\mathrm{Fe}^{2+}$ and two $\mathrm{Fe}^{3+}$ ions. Neither of the charge order patterns found here or in earlier studies $^{11,15,19,20}$ in the $P 2 / C$ or $C c$ unit cells ${ }^{18}$ fulfills this criterion. Coulomb repulsion obviously becomes less important where large screening charges are generated which minimize charge disproportionation between similar sites.

The original conjecture on charge ordering in $\mathrm{Fe}_{3} \mathrm{O}_{4}$ by Verwey and Heilmann ${ }^{35}$ conceived the charge order as localized $\mathrm{Fe}^{2+}$ and $\mathrm{Fe}^{3+}$ ions on octahedral sites. Recent experi- mental and computational studies discussed above have found that the degree of charge disproportionation, $\delta_{12}$ and $\delta_{34}$, between $\mathrm{B}$ sites is much less than a full electronic charge. However, Verwey and Heilmann ${ }^{35}$ were essentially correct in their conjecture since all conduction electrons do localize fully on octahedral B sites below $T_{V}$, although the actual charge ordering pattern is different. As far as the current authors are aware, this has not been explicitly stated in any of the recent theoretical work on $\mathrm{Fe}_{3} \mathrm{O}_{4}$.

\section{Hybrid functionals and related methods}

Since the charge order predicted by B3LYP is the same as that predicted by $\mathrm{DFT}+U$ methods, it is worth placing the former approach in context. The Kohn-Sham matrix in hybrid density functionals contains the HF exchange operator with a weighting factor. HF exchange in the HF approximation $[A=1, B=C=0$ in Eq. (1)] overestimates the band gaps of solids; predicted band gaps of solids are linearly dependent on any simple weighting factor attached to HF exchange in the exchange operator. ${ }^{30}$ It is well known that the $G W$ approximation predicts band gaps of semiconductors and insulators very well. The $G W$ approximation is essentially a dynamically screened HF theory in which the HF exchange is screened (or weighted) by the energy and wave vector dependent inverse dielectric function. The dependence of the screening of states around the band gaps in semiconductors is only weakly energy and wave vector dependent. The extent of uniform screening of the HF exchange operator in the $G W$ approximation by the "head" of the dielectric matrix is on the order of the reciprocal of the electronic dielectric constant, $1 / \boldsymbol{\epsilon}_{\infty} \sim 0.2$, for many oxides. Hence there are some similarities between hybrid density functionals and the $G W$ approximation.

Magnitudes of HF exchange matrix elements in transition metal oxides are very different for transition metal $3 d$ states and oxygen $2 p$ states. In $\mathrm{NiO}$ the $\mathrm{Ni} 3 d$ exchange energy calculated with either self-consistent HF theory or B3LYP is around $-45 \mathrm{eV}$ for majority spin states. ${ }^{54}$ In contrast, the oxygen $2 p$ exchange energy is around $-27 \mathrm{eV}$ and the exchange energy for the lowest conduction band is around $-11 \mathrm{eV}$. Inclusion of HF exchange lowers the energies of occupied states more than unoccupied states and compact states such as $d$ states are lowered more in energy and become more compact to a greater extent. $\mathrm{DFT}+U$ methods also result in greater localization of transition metal $d$ states through the $U$ term. If the parallel drawn between $G W$ and hybrid density functionals is indeed valid, then in a narrow gap oxide such as $\mathrm{Fe}_{3} \mathrm{O}_{4}$, with greater screening than a typical oxide, a smaller weight for $\mathrm{HF}$ exchange $(A=0.15$ rather than 0.20) is likely to be needed in order to account for the degree of charge disproportionation, band gap opening, and extent of bond length distortion in the charge ordered state.

\section{Phonons and vibrational spectra}

The nature of the Verwey transition has been debated over many years. ${ }^{1,4-15,21,36,55}$ While there is an abrupt change in electric conductivity at $T_{V}$, by about 2 orders of magnitude, ${ }^{1}$ other probes such as neutron diffuse scattering indicate that 
there are strong fluctuations in the cubic phase well above $T_{V}$. Neutron diffuse scattering extends up to $100 \mathrm{~K}$ above $T_{V}$ and disappears abruptly below $T_{V}{ }^{4,5}$ It has been proposed ${ }^{55}$ that the electronic and crystal structure symmetry breaking about $\mathrm{Fe} \mathrm{B}$ sites, which is observed in $\mathrm{Fe}_{3} \mathrm{O}_{4}$ below $T_{V}{ }^{8}$ is actually present above $T_{V}$. Models including existence of "molecular polarons" 5 and dynamical distortions ${ }^{55}$ which condense at $T_{V}$ have been advanced. In this scenario the Verwey transition is essentially an ordering of the lattice polarons which form about $\mathrm{Fe} \mathrm{B}$ sites with an extra electron once the thermal energy of the polarons is low enough to allow them to condense. This ordering would then be accompanied by the 2 orders of magnitude drop in conductivity which occurs on cooling through $T_{V} \cdot{ }^{1}$ In the work reported here, it has been shown that the highest frequency breathing mode phonons in $\mathrm{Fe}_{3} \mathrm{O}_{4}$ below $T_{V}$ are localized in layers. This localization may have an important part to play in the drop in conductivity at $T_{V}$.

If the evolution of IR and Raman spectra through $T_{V}$ is examined in the context of this model, the following observations can be made: linewidths of the two main IR active modes above $T_{V}$ at 350 and $600 \mathrm{~cm}^{-1}$ are fairly broad at 24 and $26 \mathrm{~cm}^{-1}$, respectively. ${ }^{42}$ These widths are large but are not much greater than linewidths in other spinels without charge order. ${ }^{56}$ Increases in linewidth on cooling through $T_{V}$ in this scenario must be due to charge ordering, which produces the splitting of frequencies found in the calculations reported above; the Raman spectrum at $125 \mathrm{~K}$ (Ref. 45) is essentially the same as the spectrum at $300 \mathrm{~K}$ in terms of the number of modes, but the linewidth of the $E_{g}$ mode around $300 \mathrm{~cm}^{-1}$ is very broad $\left(\sim 100 \mathrm{~cm}^{-1}\right)$ just above $T_{V}$ at 125 $\mathrm{K}$, suggesting a degree of disorder about $\mathrm{Fe} \mathrm{B}$ sites, and evolves into the broad band of modes between 200 and $500 \mathrm{~cm}^{-1}$ on cooling below $T_{V}$. Extra peaks in the spectrum appear abruptly between 120 and $116 \mathrm{~K}$ and are fully established at $100 \mathrm{~K}$.

The most striking finding in the work reported here is that phonons in the highest IR band and two highest Raman bands are concentrated on specific B site chains in the low temperature phase and that there are splittings in mode frequencies by $\sim 50 \mathrm{~cm}^{-1}$ for the $565 \mathrm{~cm}^{-1}$ mode $\left(540 \mathrm{~cm}^{-1}\right.$ in experiment) and by $\sim 120 \mathrm{~cm}^{-1}$ for the $584 \mathrm{~cm}^{-1}$ mode (670 $\mathrm{cm}^{-1}$ in experiment). The Raman spectrum below $T_{V}$ shows a line narrowing by $10 \mathrm{~cm}^{-1}$ (Ref. 42) and new modes appear either as distinct peaks or shoulders to strong peaks. These additional peaks may be the frequency-split peaks predicted in these calculations. Presumably one mode retains most of the Raman scattering cross section, but other frequency-split modes also have some cross section. Such a redistribution of scattering cross section may explain the observed narrowing of the band observed at $670 \mathrm{~cm}^{-1}$ by $10 \mathrm{~cm}^{-1}$ when $\mathrm{Fe}_{3} \mathrm{O}_{4}$ is cooled through $T_{V}$. Charge ordering in $\mathrm{Fe}_{3} \mathrm{O}_{4}$ has been shown to have a predominant [001] charge density modulation accompanied by a weaker $\left[00 \frac{1}{2}\right]$ modulation $;^{8}$ magnetic inelastic neutron scattering from $\mathrm{Fe}_{3} \mathrm{O}_{4}$ exhibits a $7 \mathrm{meV}$ gap in the spin wave spectrum midway to the Brillouin zone boundary for $\mathbf{q}$ vectors parallel to the $c$ axis. ${ }^{57}$ It is therefore not surprising to find strong modulations of ion displacements in phonon modes along the $c$ axis. Further experimental and theoretical work, including computed Raman cross sections, is needed to resolve the number and polarization of additional modes which are observed below $T_{V}$ around $600 \mathrm{~cm}^{-1}$.

There is one unstable mode of $A_{g}$ symmetry in the phonon calculation on $\mathrm{Fe}_{3} \mathrm{O}_{4}$ in the $P 2 / c$ unit cell. It is concentrated mainly on $\mathrm{Fe} \mathrm{A} 2$ and $\mathrm{B} 3$ and $\mathrm{O} 6$ sites and displacements are mainly parallel to the $b$ axis. The CRYSTAL package computes analytic derivatives of the total energy with respect to ion displacement $^{58,59}$ (i.e., forces on the ion including Pulay forces ${ }^{60}$ ) at several points in order to obtain numerical second derivatives of the total energy. These forces are evaluated using highly converged wave functions at each ion displacement and so the force constant matrix generated should be an excellent numerical approximation to the force constant matrix within the approximations of the B3LYP functional and computational conditions used. The phonon calculation in the $P 2 / c$ unit cell was repeated several times under different computational conditions and the unstable mode was present in each case. The instability in the $P 2 / c$ structure is likely to be caused either by the B3LYP functional or the $P 2 / c$ approximation to the full $C c$ unit cell found in experiment. ${ }^{8,49}$ The $C c$ structure is obtained from the $P 2 / c$ structure by doubling the unit cell along the $a$ and $b$ crystallographic axes. A recent DFT + Hubbard $U$ calculation, in which the crystal lattice was relaxed in both the $P 2 / c$ and $C c$ unit cells, found a different charge order for the $C c$ unit cell in sites corresponding to $\mathrm{B} 3 / \mathrm{B} 4$ chains. ${ }^{18}$ The latter charge order is similar to the charge order pattern found by refinement of resonant $\mathrm{x}$-ray diffraction studies of $\mathrm{Fe}_{3} \mathrm{O}_{4}$ (Ref. 12); the unstable mode whose ion displacements are concentrated on $\mathrm{Fe}$ and $\mathrm{O}$ sites in the $\mathrm{B} 3$ chains of the $P 2 / c$ unit cell may be an indication that the charge order found in the $P 2 / c$ unit cell is not the ground state in the $C c$ supercell.

\section{E. Verwey transition and charge transport}

The main focus of this paper is the structure and vibrational spectra of magnetite above and below $T_{V}$. In this section we consider the implications of our results for the Verwey transition itself. The relative importance and actual nature of the changes which occur in magnetite on passing through $T_{V}$ continue to be debated. A key question in this debate is whether or not a gap exists in the density of states above $T_{V}$.

Experimental evidence from photoemission and inverse photoemission has been used to argue for and against the existence of a gap above $T_{V}$. One study reported a gap of 70 meV below $T_{V}$ which closed on raising the temperature above $T_{V}{ }^{61}$; in another study a gap observed below $T_{V}$ was reduced by $50 \mathrm{meV}$ above $T_{V}$ but did not close completely. ${ }^{62}$ A more recent soft $\mathrm{x}$-ray photoemission study reported a discontinuous change in photoemission onset by $50 \mathrm{meV}$ which occurs sharply at $T_{V}{ }^{10}$ The authors of that work fitted the photoemission line shape within $1 \mathrm{eV}$ of the onset for photoemission to a small polaron Gaussian line shape. They also noted the absence of a metallic Fermi edge or conventional quasiparticle. ${ }^{10}$ Similar photoemission line shapes have been observed in a cuprate at low hole doping and the absence of a quasiparticle peak and good fit by a small polaron Gaussian 
profile were reported recently. ${ }^{63}$ The gap in the density of states of magnetite below $T_{V}$ obtained by optical conductivity measurements has been reported to be $200 \mathrm{meV}^{42}$ or 140 meV. ${ }^{53}$ A strong polaronic peak observed in the optical conductivity around $0.6 \mathrm{eV}$, when the sample is below $T_{V}$, weakens considerably above $T_{V} \cdot 42,53$

We now consider creation of small polaron charge carriers in the charge and orbitally ordered state predicted by our hybrid DFT calculations on the $P 2 / c$ structure. Creation of a small polaron hole state will occur with removal of a minority spin electron from either a B1 or B4 site while creation of a small polaron electron state will occur with addition of an electron to a B2 or B3 site. Charge transport in magnetite below $T_{V}$ is expected to occur via creation of pairs of small polaron electrons and holes and drift of these heavy carriers in an electric field. Our finding that minority spin $d$ electrons on B sites are well localized, and that this is accompanied by only a small total disproportionation of charge in agreement with experiment, ${ }^{12}$ is obviously important to the small polaron model of charge carriers in magnetite. The Verwey transition is accompanied by a structural change from a monoclinic to a cubic structure. This change in structure may be expected to cause a significant change in conductivity as small polaron electron-hole pairs are not condensed into the charge ordered ground state found below $T_{V}$.

Hybrid DFT (this work) and LDA $+U$ calculations predict a charge ordered state with charge and orbitally ordered minority spin $d$ electrons and a monoclinic crystal structure. The GGA-DFT calculations reported here predict an itinerant electron state and a cubic crystal structure. A dynamical mean field theory (DMFT) calculation performed using a density of states derived from a local spin density approximation (LSDA) calculation predicts a semiconducting state for magnetite above and below $T_{V \cdot}{ }^{23}$ The small polaron electron and hole states referred above are not included in any of these calculations as none of them includes coupling of phonons to charge carriers. The hybrid DFT methods that have been used in this work predict a small polaron state for holes introduced into a cuprate at low hole density and a polaronic optical transition at midgap. ${ }^{64}$ Work in progress on magnetite ${ }^{65}$ shows that adding electrons or holes to magnetite in hybrid DFT calculations does indeed result in small polaron states. The strongly temperature dependent conductivity in magnetite above and below $T_{V}$ is likely to be strongly associated with an activation barrier to small polaron hopping.

The GGA-DFT calculations reported here predict the crystal structure, lattice parameter, and vibrational frequencies of magnetite above $T_{V}$ in good agreement with experiment. ${ }^{8}$ However, the GGA-DFT Hamiltonian omits electron-phonon coupling which is evidently important in charge transport in magnetite and hence the itinerant electron metallic state which it predicts will not give reliable predictions of charge transport properties above $T_{V}$. A recent DMFT study of magnetite ${ }^{23}$ used the density of states derived from an LSDA calculation on the cubic magnetite structure and found that the itinerant metallic state from LSDA was replaced by two possible charge gapped states whose gaps differed by $24 \mathrm{meV} .^{23}$ These were interpreted as the states observed by photoemission and inverse photoemis- sion where the gap decreased by $50 \mathrm{meV}$ on crossing $T_{V}$ from below. ${ }^{62}$ The DMFT study did not consider the change in structure at $T_{V}$ and attributed the opening of the charge gap to Fe $d$ state electron correlations. There is abundant experimental evidence that charge carriers exist as small polarons in transition metal oxides. ${ }^{66}$ Inclusion of both electron-phonon coupling and electron correlations is evidently essential for understanding transport properties of these systems.

\section{CONCLUSION}

In conclusion, the crystal and electronic structures of $\mathrm{Fe}_{3} \mathrm{O}_{4}$ in the $F d \overline{3} m$ and $P 2 / c$ unit cells have been calculated using DFT-GGA and B3LYP hybrid density functional methods. Structure relaxations in the $P 2 / c$ unit cell using the B3LYP functional find only relatively small differences from the structure refined from $\mathrm{X}$-ray and neutron scattering data. ${ }^{8}$ Both structures are characterized by alternation in the mean $\mathrm{Fe}-\mathrm{O}$ bond length in $\mathrm{Fe} \mathrm{B}$ site octahedra along the crystalline $c$ axis. A similar modulation is found in B3LYP functional calculations reported here and in similar calculations which used a DFT-GGA $+U$ functional; ${ }^{19}$ however, both calculations predict a larger degree of modulation than was obtained from experiment. ${ }^{8}$ The modulation in crystal structure is accompanied by a charge disproportionation between B1/B2 and B3/B4 octahedral Fe sites. Bond valence sums computed from the experimental crystal structure imply a difference in valence of the Fe ions at these sites of 0.2 , much less than the difference between their formal valences $(+2 /+3)$. Estimates of charge disproportionation based on GGA-DFT $+U$, SICDFT, or B3LYP functional calculations range from $0.20 e$ (Ref. 18) to $0.36 e$ (Ref. 14); the B3LYP calculation predicts a disproportionation of $\delta_{12}=0.18 e$ and $\delta_{34}=0.14 e$. The minority spin valence band contains an isolated group of eight bands at the valence band maximum. Electrons in these bands are strongly localized on Fe B1 and B4 sites, showing that conduction electrons are indeed localized on specific $\mathrm{Fe}$ octahedral sites formally as $\mathrm{Fe}^{2+}$ or $\mathrm{Fe}^{3+}$, but with a different ordering pattern from that originally envisaged by Verwey.

Vibrational modes of $\mathrm{Fe}_{3} \mathrm{O}_{4}$ in the cubic high temperature $F d \overline{3} m$ and monoclinic low temperature $P 2 / c$ unit cells have been computed using DFT-GGA and B3LYP hybrid DFT functionals, respectively. There is good agreement between IR and Raman active mode frequencies for the $F d \overline{3} m$ phase with a $30.9 \mathrm{~cm}^{-1}$ mean absolute difference between experimental mode frequencies and predicted frequencies. The IR conductivity for the $P 2 / c$ phase was calculated using a Lorentz oscillator model and eigenmodes calculated using a B3LYP functional. When B3LYP mode frequencies are scaled down by $5 \%$ there is good agreement between peak positions in the LO model and experiment. Increases in IR linewidth from 24 to 39 and from 26 to $36 \mathrm{~cm}^{-1}$ are observed in experiment on cooling through $T_{V \cdot}{ }^{42}$ This may be explained by the appearance of a number of new IR active modes as the symmetry is broken on going from the $F d \overline{3} m$ to the $P 2 / c$ structure.

There are major changes in the Raman spectrum on cooling through $T_{V}$. Raman active mode densities at the $\Gamma$ point 


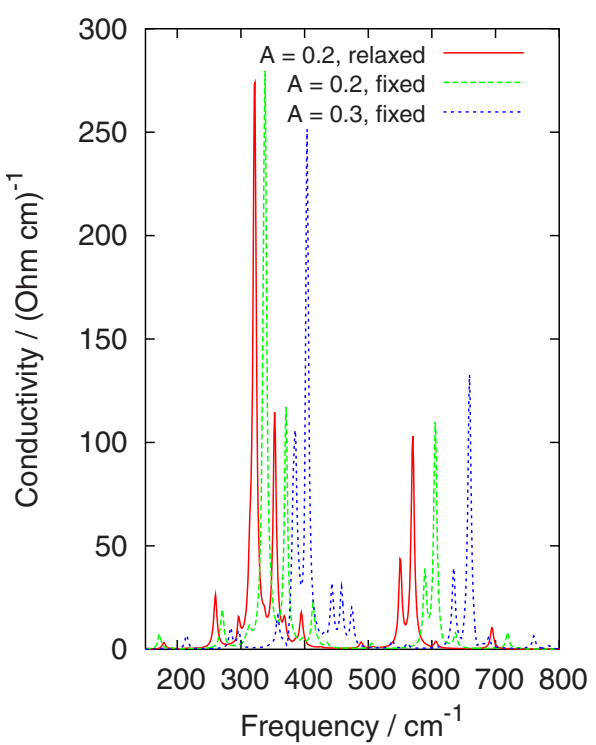

FIG. 16. (Color online) Conductivity of $\mathrm{Fe}_{3} \mathrm{O}_{4}$ parallel to the $c$ axis computed using a B3LYP functional and the LO model in Eq. (2) with lattice constants fixed at experimental values or fully relaxed and with weights of HF exchange, $A=0.2$ or 0.3 .

of appropriate symmetry are compared to polarized Raman spectra. There is good agreement in distributions of mode frequencies for both $A_{g}$ and $B_{g}$ modes when compared to polarized Raman spectra in experimental $X X$ and $X Y$ geometries, except for the $X Y$ spectrum below $300 \mathrm{~cm}^{-1}$. In the $F d \overline{3} m$ structure the two most intense lines are found at 540 $\left(T_{2 g}\right)$ and at $670 \mathrm{~cm}^{-1}\left(A_{g}\right)$. In the $P 2 / c$ structure each of these modes is replaced by $4 A_{g}$ and $4 B_{g}$ Raman active modes which are split in frequency by 50 or $100 \mathrm{~cm}^{-1}$. This splitting occurs because charge ordering, which accompanies the transition between the cubic $F d \overline{3} m$ and monoclinic $P 2 / c$ structures, induces symmetry breaking in force constants on Fe octahedral B sites. A detailed comparison of Raman line positions and intensities from theory and experiment would provide a powerful probe of the Verwey transition.

\section{ACKNOWLEDGMENTS}

The authors are grateful to David Tanner for helpful discussions and for use of IR data on magnetite reproduced from Fig. 12 of Ref. 42. C.H.P. acknowledges discussions with Paul Attfield on X-ray and neutron scattering work in Ref. 8 and with Roberto Dovesi on choices of computational parameters. This work was supported by the Irish Higher Education Authority (IHEA) under Program No. PRTLI3IITAC2. Computer time was provided by the Trinity Centre for High Performance Computing which is supported by the IHEA, Science Foundation Ireland, and the Irish National
Development Plan. L.V.G. acknowledges support by the National Science Foundation under Grant No. DMR-08-05-073, Research Corporation Cottrell College Science under Grant No. CC 6130, Petroleum Research Fund under Grant No. 40926-GB10, and Office of Naval Research (ONR) under Grant No. N00014-06-1-0133.

\section{APPENDIX: DETAILS OF CALCULATIONS}

All electron Gaussian orbital basis sets used in this work are an Fe $20 s 12 p 6 d$ basis contracted to $5 s 4 p 2 d$, previously used to study hematite ${ }^{67}$ and a $14 s 6 p$ basis contracted to $4 s 3 p$, previously used to study $\mathrm{NiO} .{ }^{68}$ Integration over the Brillouin zone was done using $4 \times 4 \times 4$ or $6 \times 6 \times 6$ (phonon calculations only) Monkhorst-Pack nets ${ }^{69}$ for the $P 2 / c$ unit cell and a $6 \times 6 \times 6$ net for the $F d \overline{3} m$ unit cell. Lattice sum tolerances of 777714 and the densest standard grid for integration in the DFT part of the Hamiltonian in the CRYSTAL package were used. Figure 2 was produced using the XCRYSDEN visualization package ${ }^{70}$ and phonon eigenvectors were drawn using the MOLDRAW package. ${ }^{71}$

Vibrational modes of $\mathrm{Fe}_{3} \mathrm{O}_{4}$ at the $\Gamma$ point of the Brillouin zone were calculated using the CRYSTAL package ${ }^{48,58}$ and the B3LYP hybrid functional. ${ }^{72,73}$ Vibrational frequencies of the magnetic insulator, andradite $\mathrm{Ca}_{3} \mathrm{Fe}_{2} \mathrm{Si}_{3} \mathrm{O}_{12}$, calculated using the CRYSTAL package, have a mean absolute error less than $10 \mathrm{~cm}^{-1}$ (Ref. 74) when compared to experiment. A study of the relative accuracies of HF, B3LYP, local density approximation (LDA) and Perdew-Burke-Enzerhof (PBE) functionals for calculating vibrational mode frequencies of $\alpha$ quartz at the $\Gamma$ point found mean absolute errors of $44,5.8,17.5$, and $21.0 \mathrm{~cm}^{-1}$ and maximum absolute errors of 85.2, 22.1, 42.6 , and $40.1 \mathrm{~cm}^{-1}$, respectively. ${ }^{58}$ The mean absolute error in the phonon calculation on $F d \overline{3} m \mathrm{Fe}_{3} \mathrm{O}_{4}$ presented here was $30.9 \mathrm{~cm}^{-1}$ and the maximum absolute error was $86 \mathrm{~cm}^{-1}$ for the $A_{g}$ mode. This breathing mode is predicted to lie at a very similar frequency by another DFT-GGA calculation, ${ }^{22}$ whereas frequencies predicted by B3LYP for equivalent modes in the $P 2 / c$ unit cell are much closer to the experimental value of $670 \mathrm{~cm}^{-1}$. These larger errors likely reflect the more complex electronic structure of a narrow gap or metallic oxide compared with quartz, which is a wide gap insulator. Magnitudes of frequencies of modes which correspond to lattice translations were around $1 \mathrm{~cm}^{-1}$.

The effect of lattice constant and weight of HF exchange in the functional on vibrational frequencies is illustrated in Fig. 16. There is a small downward shift in peaks in the conductivity spectrum when lattice constants are relaxed and there are only minor changes in the shape of the spectrum. Increasing the weight of HF exchange in the functional shifts frequencies upward by about $10 \%$. 
${ }^{1}$ E. J. W. Verwey, Nature (London) 144, 327 (1939).

${ }^{2}$ E. J. W. Verwey and P. W. Haayman, Physica 8, 979 (1941).

${ }^{3}$ P. W. Anderson, Phys. Rev. 115, 2 (1959).

${ }^{4}$ S. M. Shapiro, M. Iizumi, and G. Shirane, Phys. Rev. B 14, 200 (1976).

${ }^{5}$ Y. Yamada, N. Wakabayashi, and R. M. Nicklow, Phys. Rev. B 21, 4642 (1980).

${ }^{6}$ J. M. Zuo, J. C. H. Spence, and W. Petuskey, Phys. Rev. B 42, 8451 (1990).

${ }^{7}$ P. Novák, H. Stěpánková, J. Englich, J. Kohout, and V. A. M. Brabers, Phys. Rev. B 61, 1256 (2000).

${ }^{8}$ J. P. Wright, J. P. Attfield, and P. G. Radaelli, Phys. Rev. B 66, 214422 (2002).

${ }^{9}$ R. J. Goff, J. P. Wright, J. P. Attfield, and P. G. Radaelli, J. Phys.: Condens. Matter 17, 7633 (2005).

${ }^{10}$ D. Schrupp, M. Sing, M. Tsunekawa, H. Fujiwara, S. Kasai, A. Sekiyama, S. Suga, T. Muro, V. A. M. Brabers, and R. Claessen, Europhys. Lett. 70, 789 (2005).

${ }^{11}$ D. J. Huang et al., Phys. Rev. Lett. 96, 096401 (2006).

${ }^{12}$ E. Nazarenko, J. E. Lorenzo, Y. Joly, J. L. Hodeau, D. Mannix, and C. Marin, Phys. Rev. Lett. 97, 056403 (2006).

${ }^{13}$ G. K. Rozenberg, M. P. Pasternak, W. M. Xu, Y. Amiel, M. Hanfland, M. Amboage, R. D. Taylor, and R. Jeanloz, Phys. Rev. Lett. 96, 045705 (2006).

${ }^{14}$ Z. Szotek, W. M. Temmerman, A. Svane, L. Petit, G. M. Stocks, and H. Winter, Phys. Rev. B 68, 054415 (2003).

${ }^{15}$ H.-T. Jeng, G. Y. Guo, and D. J. Huang, Phys. Rev. Lett. 93, 156403 (2004).

${ }^{16}$ I. Leonov, A. N. Yaresko, V. N. Antonov, M. A. Korotin, and V. I. Anisimov, Phys. Rev. Lett. 93, 146404 (2004).

${ }^{17}$ G. K. H. Madsen and P. Novak, Europhys. Lett. 69, 777 (2005).

${ }^{18}$ H.-T. Jeng, G. Y. Guo, and D. J. Huang, Phys. Rev. B 74, 195115 (2006).

${ }^{19}$ H. P. Pinto and S. D. Elliott, J. Phys.: Condens. Matter 18, 10427 (2006).

${ }^{20}$ I. Leonov, A. N. Yaresko, V. N. Antonov, and V. I. Anisimov, Phys. Rev. B 74, 165117 (2006).

${ }^{21}$ P. Piekarz, K. Parlinski, and A. M. Oles, Phys. Rev. Lett. 97, 156402 (2006).

${ }^{22}$ P. Piekarz, K. Parlinski, and A. M. Oles, Phys. Rev. B 76, 165124 (2007).

${ }^{23}$ L. Craco, M. S. Laad, and E. Müller-Hartmann, Phys. Rev. B 74, 064425 (2006).

${ }^{24}$ M. Iizumi and G. Shirane, Solid State Commun. 17, 433 (1975).

${ }^{25}$ J. Yoshida and S. Iida, J. Phys. Soc. Jpn. 47, 1627 (1979).

${ }^{26}$ P. A. M. Dirac, Proc. Cambridge Philos. Soc. 26, 376 (1930).

${ }^{27}$ A. D. Becke, Phys. Rev. A 38, 3098 (1988).

${ }^{28}$ C. Lee, W. Yang, and R. G. Parr, Phys. Rev. B 37, 785 (1988).

${ }^{29}$ S. H. Vosko, L. Wilk, and M. Nusair, Can. J. Phys. 58, 1200 (1980).

${ }^{30}$ F. Cora, M. Alfredsson, G. Mallia, D. S.Middlemiss, W. C. Mackrodt, R. Dovesi, and R. Orlando, Struct. Bonding (Berlin) 113, 171 (2004).

${ }^{31}$ L. Hedin, Phys. Rev. 139, A796 (1965).

${ }^{32}$ F. Aryasetiawan and O. Gunnarsson, Rep. Prog. Phys. 61, 237 (1998).

${ }^{33}$ J. P. Wright, J. P. Attfield, and P. G. Radaelli, Phys. Rev. Lett. 87, 266401 (2001).

${ }^{34}$ A. J. M. Kuipers and V. A. M. Brabers, Phys. Rev. B 20, 594 (1979).
${ }^{35}$ E. J. W. Verwey and E. L. Heilmann, J. Chem. Phys. 15, 174 (1947).

${ }^{36}$ P. W. Anderson, Phys. Rev. 102, 1008 (1956).

${ }^{37}$ G. Subias, J. Garcia, J. Blasco, M. Grazia Proietti, H. Renevier, and M. Concepcion Sanchez, Phys. Rev. Lett. 93, 156408 (2004).

${ }^{38}$ R. D. Waldron, Phys. Rev. 99, 1727 (1955).

${ }^{39}$ W. B. White and B. A. DeAngelis, Spectrochim. Acta, Part A 23, 985 (1967).

${ }^{40}$ J. L. Verble, Phys. Rev. B 9, 5236 (1974).

${ }^{41}$ L. Degiorgi, I. Blatter-Mörke, and P. Wachter, Phys. Rev. B 35 , 5421 (1987).

${ }^{42}$ L. V. Gasparov, D. B. Tanner, D. B. Romero, H. Berger, G. Margaritondo, and L. Forró, Phys. Rev. B 62, 7939 (2000).

${ }^{43}$ C. M. Julien and M. Massot, Mater. Sci. Eng., B 97, 217 (2003).

${ }^{44}$ O. N. Shebanova and P. Lazor, J. Solid State Chem. 174, 424 (2003).

${ }^{45}$ L. V. Gasparov, D. Arenas, K.-Y. Choi, G. G'untherodt, H. Berger, L. Forro, G. Margaritondo, V. V. Struzhkin, and R. Hemley, J. Appl. Phys. 97, 10A922 (2005).

${ }^{46}$ B. Handke, A. Kozlowski, K. Parlinski, J. Pzrzewoznik, T. Slezak, A. I. Chumakov, L. Niesen, Z. Kakol, and J. Korecki, Phys. Rev. B 71, 144301 (2005).

${ }^{47}$ J. P. Perdew and Y. Wang, Phys. Rev. B 45, 13244 (1992).

${ }^{48}$ R. Dovesi et al., Crystal06 User's Manual (University of Torino, Torino, 2007).

${ }^{49}$ M. Iizumi, T. F. Koetzle, G. Shirane, S. Chikazumi, M. Matsui, and S. Todo Acta Crystallogr., Sect. B: Struct. Crystallogr. Cryst. Chem. 38, 2121 (1982).

${ }^{50}$ E. J. W. Verwey, P. W. Haayman, and F. C. Romeijn, J. Chem. Phys. 15, 181 (1947).

${ }^{51}$ P. Graves, C. Johnston, and J. Campaniello, Mater. Res. Bull. 23, 1651 (1988).

${ }^{52}$ See EPAPS document No. E-PRBMDO-79-073916 for phonon eigenvectors with (i) largest optical activity in the band between 300 and 400 wave numbers and (ii) Raman activity and $B_{g}$ symmetry in the band between 560 and 640 wave numbers. For more information on EPAPS, see http://www.aip.org/pubservs/ epaps.html.

${ }^{53}$ S. K. Park, T. Ishikawa, and Y. Tokura, Phys. Rev. B 58, 3717 (1998).

${ }^{54}$ C. H. Patterson, Int. J. Quantum Chem. 106, 3383 (2006).

${ }^{55}$ G. Subías, J. García, and J. Blasco, Phys. Rev. B 71, 155103 (2005).

${ }^{56}$ A. M. Hofmeister, Am. Mineral. 86, 1188 (2001).

${ }^{57}$ R. J. McQueeney, M. Yethiraj, W. Montfrooij, J. S. Gardner, P. Metcalf, and J. M. Honig, Phys. Rev. B 73, 174409 (2006).

${ }^{58}$ F. Pascale, C. Zicovich-Wilson, F. Lopez, B. Civalleri, R. Orlando, and R. Dovesi, J. Comput. Chem. 25, 888 (2004).

${ }^{59}$ C. Zicovich-Wilson, F. Pascale, C. Roetti, V. Saunders, R. Orlando, and R. Dovesi, J. Comput. Chem. 25, 1873 (2004).

${ }^{60}$ P. Pulay, Mol. Phys. 17, 197 (1969).

${ }^{61}$ A. Chainani, T. Yokoya, T. Morimoto, T. Takahashi, and S. Todo, Phys. Rev. B 51, 017976 (1995).

${ }^{62}$ J. H. Park, L. H. Tjeng, J. W. Allen, P. Metcalf, and C. T. Chen, Phys. Rev. B 55, 12813 (1997).

${ }^{63}$ K. M. Shen, F. Ronning, W. Meevasana, D. H. Lu, N. J. C. Ingle, F. Baumberger, W. S. Lee, L. L. Miller, Y. Kohsaka, M. Azuma, M. Takano, H. Takagi, and Z. X. Shen, Phys. Rev. B 75, 075115 (2007). 
${ }^{64}$ C. H. Patterson, Phys. Rev. B 77, 094523 (2008).

${ }^{65}$ A. Chakrabarty and C. H. Patterson (unpublished).

${ }^{66}$ A. M. Stoneham, J. Gavartin, A. L. Schluger, A. V. Kimmel, D. M. Ramo, H. M. Ronnow, G. Aeppli, and C. Renner, J. Phys.: Condens. Matter 19, 255208 (2007).

${ }^{67}$ M. Catti, G. Valerio, and R. Dovesi, Phys. Rev. B 51, 7441 (1995).

${ }^{68}$ M. D. Towler, N. L. Allan, N. M. Harrison, V. R. Saunders, W. C. Mackrodt, and E. Aprà, Phys. Rev. B 50, 5041 (1994).
${ }^{69}$ H. Monkhorst and J. D. Pack, Phys. Rev. B 13, 5188 (1976).

${ }^{70}$ A. Kokalj, Comput. Mater. Sci. 28, 155 (2003).

${ }^{71}$ P. Ugliengo, D. Viterbo, and G. Borzani, J. Appl. Crystallogr. 21, 75 (1988).

${ }^{72}$ A. D. Becke, J. Chem. Phys. 98, 5648 (1993).

${ }^{73}$ P. J. Stephens, F. J. Devlin, C. F. Chabalowski, and M. J. Frisch, J. Phys. Chem. 98, 11623 (1994).

${ }^{74}$ F. Pascale, M. Catti, A. Damin, R. Orlando, V. Saunders, and R. Dovesi, J. Phys. Chem. B 109, 18522 (2005). 